\title{
Théocrite : Idylle 7
}

\section{Laurence Plazenet}

\section{Résumé}

L'idylle 7 de Théocrite procède à la mise en scène d'une initiation poétique. Ses protagonistes sont en fait des complices, qui s'emploient à dénoncer une lecture littérale des figures et du discours de la poésie bucolique, fondée sur la dissonance, le mélange des genres, l'humour. Simultanément, les Thalysies manifestent une réflexion sur le statut et le travail du poète bucolique, qui allie renouveau et exploitation de la tradition. Le patronage jusque-là concurrent de Pan et des Muses trouve ainsi une résolution harmonieuse dans celui , emblématique, de Déméter. Une lecture suivie de l'idylle 7 permet de rendre compte de la logique profonde d'un texte qui s'avère une véritable poétique en action.

\section{Citer ce document / Cite this document :}

Plazenet Laurence. Théocrite : Idylle 7. In: L'antiquité classique, Tome 63, 1994. pp. 77-108;

doi : https://doi.org/10.3406/antiq.1994.1184

https://www.persee.fr/doc/antiq_0770-2817_1994_num_63_1_1184

Fichier pdf généré le 07/04/2018 


\section{Théocrite : idylle 7}

La structure narrative de l'idylle 7 de Théocrite, Les Thalysies, suggère une lecture du texte comme art poétique. Au début du poème, le narrateur annonce qu'il se rend, accompagné d'Eucritos et Amyntas, chez Phrasidémos et Antigénès, pour célébrer les Thalysies. En route, il rencontre Lycidas et chacun chante à son tour un poème bucolique. Puis ils se séparent et Simichidas conclut sur l'évocation de la félicité goûtée chez ses hôtes. La composition circulaire de l'idylle met en valeur l'échange des chants bucoliques entre Lycidas et Simichidas, situé au centre. Cet épisode est en outre encadré par le don que Lycidas fait de sa houlette à Simichidas, réminiscence ou réécriture du début de la Théogonie (v. 22 à 31).

L'idylle 7 a longtemps été interprétée comme la mise en scène d'une expérience personnelle, l'initiation poétique du jeune Théocrite par un aîné dont la présentation, voisine de celle des divinités dans l'Iliade, l'Odyssée et plus encore Hésiode, suggère discrètement qu'il s'agit peut-être d'Apollon lui-même'. La fin du poème, sa beauté, tiennent lieu d'illustration de la perfection poétique désormais atteinte par Simichidas. Cependant, une étude détaillée de l'idylle 7 fait saillir nombre de contrastes et de contradictions. Plusieurs travaux récents, sensibles à la subtilité des Thalysies, ont renoncé à cette vision univoque, sans proposer aucune autre interprétation globale du poème ${ }^{2}$.

1 Voir M. PUElma, Die Dichterbegegnung in Theokrits Thalysien, dans M.H., 17 (1960), p. 147-149 ; A. CAMERON, The form of the Thalysia, dans Miscellanea di Studi alessandrini in memoria di Augusto Rostagni, Torino, 1963, p. 291-307 ; G. GIANGRANDE, Théocrite, Simichidas et les Thalysies, dans A.C., 37 (1968), p. 515-531; F. WILliAMS, A Theophany in Theocritus, dans C.Q., 21 (1971), p. 137-145, discuté par E. L. BowIE, Theocritus' seventh idyll, dans C.Q., 35 (1985), p. 67-91.

2 Voir les conclusions de G. O. HuTCHINSON sur l'idylle 7 dans Hellenistic Poetry. Theocritus, Oxford, Clarendon Press, 1988, p. 212. Il pose alors : «One result of this part of the chapter has been to remove the effect of Theocritus' individual poems from a significance conferred by supposed debates of the time, and also from a significance conferred by each other. In this sense, the individual poem is strongly isolated. Unlike Callimachus, Theocritus does not build his short poems into larger structures ; he does not encompass, acquires a vigorous and striking individuality, not least through the production and exploitation of marked difference within the poem in its levels of narrative and its level of emotion.». C. SEGAL, Theocritus' Seventh Idyll and Lycidas, dans Poetry and Myth in Ancient Pastoral, Princeton, Princeton University Press, 1981, p. 7, en arrive à définir les poètes hellénistiques comme «bold collagists of heterogeneous fragments», tandis que S. GoldHILl, The Poet's Voice. Essay on Poetics and Greek 
Mais n'est-il pas possible d'intégrer ces éléments dans une analyse cohérente de l'idylle 7 , en procédant à une explication plus littérale de son texte ? C'est que nous allons tenter.

Lycidas et Simichidas sont d'ordinaire considérés comme des personnages antithétiques ${ }^{3}$. Leur confrontation devient alors emblématique $^{4}$, opposant le chevrier-poète en contact avec la nature et les dieux au jeune poète citadin, ambitieux et maladroit. Peut-être est-ce faire bon marché d'une ironie qui ne permet pas de maintenir semblable répartition des rôles. Lycidas est décrit par Simichidas et Simichidas par Lycidas : chacun des deux charge le portrait de l'autre.

L'ironie domine la présentation des deux protagonistes de l'idylle 7. Dans le cas de Lycidas, l'insistance de la description physique constitue un signal adressé à la méfiance du lecteur. En deux vers

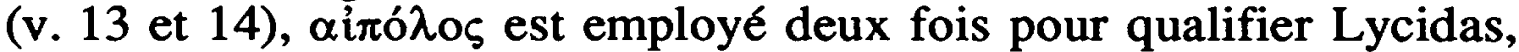

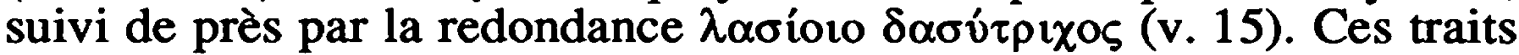
sont trop appuyés, dans une poésie par ailleurs si habile, pour être ignorés ou portés au compte de l'inattention du poète. Ainsi la comparaison du portrait de Lycidas avec les autres évocations de chevriers dans le corpus de Théocrite fait ressortir la spécificité de l'idylle 7. Aucun texte ne procède à une description systématique ${ }^{5}$, pas même l'idylle 6 qui possède une introduction narrative. Le lecteur dispose tout au plus d'informations disséminées dans un dialogue ou

Literature, Cambridge, Cambridge University Press, 1991, p. 243, cite R. BARTHES pour conclure, de la fragmentation qu'il distingue chez Théocrite, sur l'idée d'une composition rhapsodique.

3 D. M. HALPERIN, Before Pastoral : Theocritus and the Ancient Tradition of Bucolic Poetry, New Haven, Yale University Press, 1983, Bucolic and Pastoral in Theocritus, p. 120, qualifie Lycidas de «quintessential goatherd». C. SEGAL parle de Lycidas comme du «goatherd par excellence» dans op. cit., p. 123, faisant écho à l'affirmation plus ancienne de G. GIANGRANDE, op. cil., p. $515:$ : (...) Lycidas -un pâtre à toute épreuve, décrit avec un réalisme heureux par Théocrite», qui renvoie lui-même à l'analyse de Ph.-E. LEGRAND, Étude sur Théocrite, Paris, 1898 (Bibliothèques des Écoles Françaises d'Athènes et de Rome, 79), p. 153.

4 Voir, parmi les ouvrages les plus récents : E. L. BowIE, op. cit.; S. GoldHILL, Framing and Polyphony : Readings in Hellenistic Poetry, dans P.C.P.S., 32 (1986), p. $25-42$ et The Poet's Voice. Essay... (op. cit.) ; D. M. HALPERIN, op. cit. ; G. O. HuTCHINSON, op. cit. ; C. SEgal, Theocritus' Seventh Idyll and Lycidas, dans W.S., Neue Folge, 8 (1974), p. 20-76 et Simichidas' Modesty: Theocritus' Idyll 7.44, dans A.J.Ph., 95 (1974), p. 128-136, réditées dans Poetry and Myth... (op. cit.), p. 110-166 et p. 167-175. G. GIANGRANDE, op. cit., p. 521-523, utilise la compétence de Lycidas pour montrer que le chevrier dément les prétentions poétiques de Simichidas.

5 Voir idylles 1, 4, 5, 6, 10. Dans l'idylle 11, le Cyclope, Polyphème ébauche un autoportrait lorsqu'il attribue à son physique les esquives de Galatée. Il n'est pas volontaire comme celui que Simichidas fait de Lycidas. Il releve en revanche les ridicules du personnage et procède par allusions, par comparaisons implicites. 
des apostrophes. Ce type de caractérisation correspond à ce qu'on trouve non seulement dans la Théogonie ${ }^{6}$, quand la Muse s'adresse aux pâtres en général à travers Hésiode, mais aussi dans l'Odyssée, lorsque est évoqué Eumée?.

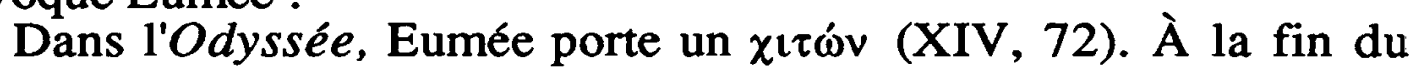
chant, il revêt une houppelande $(\chi \lambda \alpha \hat{\imath} v \alpha, X I V, 528)$ et une peau de chèvre ( $\left.\alpha_{\alpha} \alpha \eta, X I V, 530\right)$ pour se protéger du vent et du froid lorsqu'il sort passer la nuit près de son troupeau. Le texte de l'Odyssée insiste sur la rigueur du temps, qui justifie pareil équipage. Hésiode, dans les Travaux et les Jours, préconise un accoutrement semblable à celui d'Eumée, quand il indique au paysan comment se vêtir pour affronter les intempéries de l'hiver ${ }^{8}$. Contre la pluie, il conseille de porter un manteau imperméable composé de peaux de chevreaux cousues ensemble ${ }^{9}$. C'est le type de vêtement que Lycidas utilise dans l'idylle 7. La redondance $\lambda \alpha \sigma i ́ o t o ~ \delta \alpha \sigma v \tau \rho \imath \chi o \zeta ~(v .15)$ insiste même sur son épaisseur. Toutefois la scène se déroule en plein midi, au plus fort de la chaleur, quand les lézards cèdent à la torpeur ${ }^{10}$. La notation est donc invraisemblable et ironique.

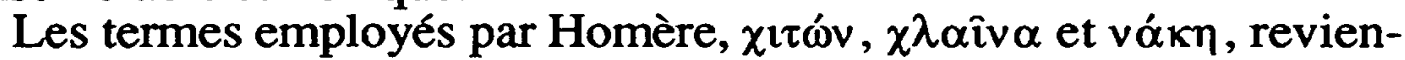
nent dans plusieurs idylles de Théocrite ${ }^{11}$, mais pas dans l'idylle 7 . Elle

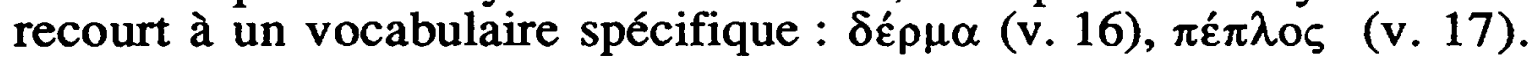
Le nom $\delta \varepsilon ́ \rho \mu \alpha$ a une valeur ironique : par référence à Hésiode, qui désigne ainsi le manteau de peaux dont il parle, mais aussi dans le contexte interne des Idylles. Les deux seuls poèmes dans lesquels $\delta \varepsilon ́ \rho \mu \alpha$ désigne une peau de bête portée comme vêtement sont d'attribution incertaine. Il s'agit des idylles 22 , les Dioscures ${ }^{12}$, et 25 , Héraclès tueur de lion ${ }^{13}$. La peau de bête mentionnée appartient alors à la panoplie d'Amycos et d'Héraclès, qui sont des personnages de style épique. Ils sont revêtus, de façon symbolique, de peaux de lion : comparés à eux, Lycidas et sa peau de bouc apparaissent sous un jour humoristique et sont en rupture avec le reste des personnages du monde bucolique. Chez Homère et Hésiode, il est question de peaux de chèvre ou de chevreaux. La peau de bouc de Lycidas, impropre à la saison et caricaturale, a tout du déguisement. La mention du $\pi \varepsilon ́ \pi \lambda \circ \varsigma$ aussi possède une valeur comique. En effet, il s'agit d'un vêtement ample,

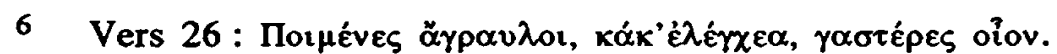

7 Chants XIV, XV, XVI, XVII, XX, XXII. Le personnage de Mélanthios, quant à lui, n'est jamais décrit.

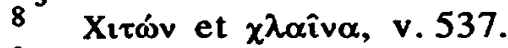

9 Théogonie, v. 544.

10 D'après Lycidas lui-même, v. 21-23.

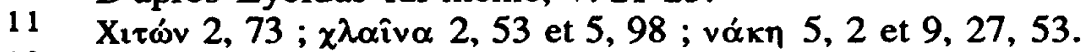

12 Vers 52.

13 Vers 63, 175 et 277.
} 
voire de cérémonie, qui ne convient aucunement aux travaux des champs ${ }^{14}$. Dans Homère, Eumée retroussait encore son $\chi \imath \tau \omega ́ v$, bien qu'il fût court, pour se mouvoir plus librement. L'ajout de l'adjectif $\gamma \varepsilon$ $\rho \omega v$, pour qualifier le $\pi \dot{\varepsilon} \pi \lambda{ }_{0} \varsigma$ de Lycidas, ne contredit pas l'idée que cette tenue n'est pas celle d'un chevrier. La vétusté du vêtement ne le rend pas plus pratique. Elle suggère un misérabilisme de comédie. En

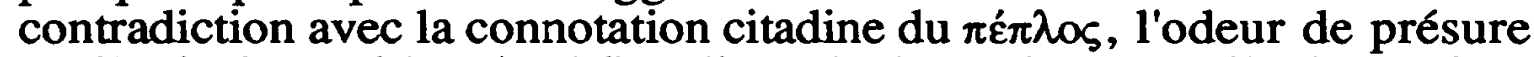
attribuée à Lycidas (v. 16) relève de la caricature : il n'en existe d'emploi similaire dans aucune autre idylle. De surcroît, quand

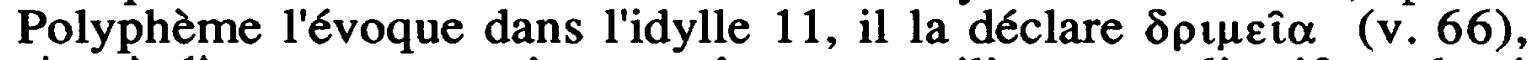
c'est-à-dire «perçante, piquante, âcre», en utilisant un adjectif employé par Homère pour qualifier traits, batailles ou colère. L'odeur de la présure est désignée à l'attention comme quelque chose de particulièrement abominable, même pour Polyphème.

On observe ainsi une dénonciation constante du personnage typique de chevrier que Lycidas serait censé incarner. Son statut insolite est renforcé par la juxtaposition d'un vocabulaire dont les Idylles ${ }^{15}$ ne révèlent pas d'autre exemple. Comme son costume, le discours de Lycidas dénonce l'ambiguîté de son personnage, puisqu'il emploie, dans le passage où il évoque Simichidas, un vocabulaire souvent rare et chargé de connotations littéraires ${ }^{16}$. C'est une façon de manifester la contradiction entre le véritable Lycidas, révélé par ses références ou sa culture, et la grossièreté des apparences qui lui sont attribuées.

Il n'est pas non plus possible de prétendre voir en Simichidas le portrait pris au vif d'un jeune auteur ambitieux et naïf. Cette lecture du personnage dépend essentiellement de la première image qu'en donne Lycidas (v. 21-26). Or, ce passage a toutes les caractéristiques d'une scène d'exposition dramatique. En l'espace de six vers, Lycidas indique le nom de son interlocuteur, le moment auquel se déroule la rencontre, procède à une rapide évocation du lieu où elle intervient et inclut quelques didascalies dans son propos ${ }^{17}$. La densité et l'aspect caractéristique du passage invitent le lecteur à considérer dans cette évocation de Simichidas la construction d'une persona. Lycidas emploie à cette

$14 \Pi \dot{n} \pi \lambda o \zeta$ est encore employé par Théocrite dans l'idylle 28, la Quenouille (v. 10), pour désigner les vêtements d'hommes dont la quenouille, offerte à la femme d'un médecin de Milet, filera la laine. Mis en parallèle avec les «étoffes fluides» dont seront faites les robes des femmes, $\pi \dot{\varepsilon} \pi \lambda_{0 \zeta}$ désigne manifestement une tenue élégante.

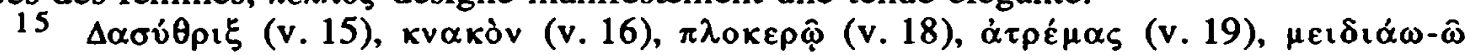
(v. 20), $\gamma \dot{\varepsilon} \lambda \omega \varsigma$ (v. 20).

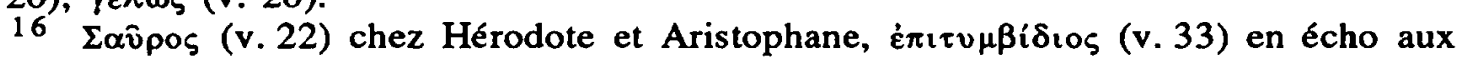
Oiseaux, (v. 475), кopv $\delta \alpha \lambda \lambda i \delta \varepsilon s$ (v. 23) chez Simonide, $\dot{\eta} \lambda \alpha i v \omega$ (v. 23) avec un sens

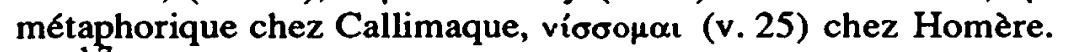

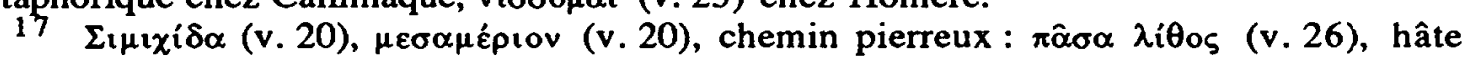

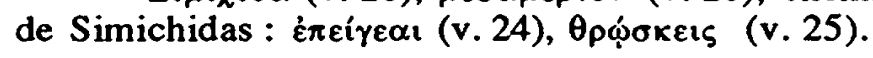


occasion un vocabulaire souvent sans autre écho dans les Idylles ${ }^{18}$ et de tonalité poétique. Concentré sur des mots qui dressent le décor de la scène, ce choix montre clairement qu'elle se situe dans un univers littéraire. Le personnage de Simichidas dont Lycidas brosse le portrait n'a d'existence que par référence à un discours poétique et non par rapport à une quelconque réalité.

On observe en outre la reprise de procédés qui apparaissaient dans la description de Lycidas par Simichidas. Le seul détail physique que Lycidas livre sur Simichidas concerne ses bottes. Il est chaussé d'ó $\rho \beta v \lambda i \delta \varepsilon \varsigma$ (v. 26). Il s'agit de demi-bottes portées par les campagnards, les chasseurs ou les voyageurs. Simichidas n'a qu'un court chemin à parcourir ${ }^{19}$ : l'idée qu'il porte des chaussures de voyage le rend ridicule. Mais il est probable que Lycidas fait également référence, par ce détail insolite, à la scène de l'Odyssée où Ulysse aperçoit Eumée pour la première fois. Eumée est occupé à se tailler des bottes ${ }^{20}$. Dans une séquence qui veut présenter un personnage typique, cette occupation prend une valeur aussi exemplaire que la description de la porcherie construite par Eumée ${ }^{21}$. Lycidas s'amuse donc à pourvoir Simichidas d'un attribut qui vient contredire son statut de prétendu citadin. On note même un renchérissement de l'auteur, qui transforme les $\pi \dot{\varepsilon} \delta \imath \lambda \alpha$ d'Eumée et du paysan d'Hésiode ${ }^{22}$, qui ont une valeur générale, en $\dot{\alpha} \rho \beta v \lambda i ́ \delta \varepsilon s$, dont la connotation rustique est très accentuée.

Une interprétation réaliste des personnages de Lycidas et de Simichidas est impossible. Aucun des deux portraits ne permet de définir l'identité ni le statut exact de son sujet. L'ironie s'exerçant à travers des amplifications et des contradictions qui portent sur l'image du personnel de la poésie bucolique, implique une distance et un jeu sur ces représentations elles-mêmes.

Loin de contredire la présentation ironique que chacun des protagonistes fait de l'autre, leurs propres interventions sont modelées de façon à renforcer l'incertitude. Dans le poème qu'il récite (v. 52-89), Lycidas se met deux fois en scène : dans un premier tableau (v. 6373), il boit à la santé de son amant et écoute des chansons, dans une attitude familière de la tradition symposiaque, liée à un contexte

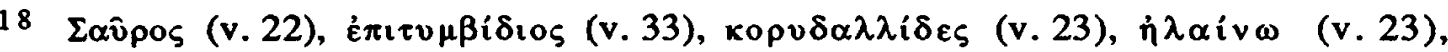

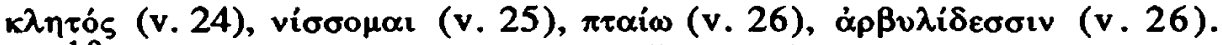

19 Voir E. L. BowIE, op. cit., p. 76, n. 45.

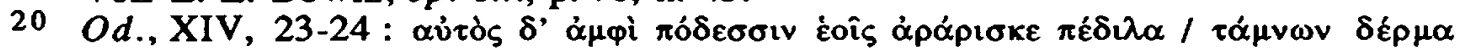
Bóecov घ̇üxpoés.

21 Od., XIV, 5-22.

22 Les Travaux et les Jours, v. 541. 
urbain ${ }^{23}$. Puis, il imagine qu'il garde les chèvres de Comatas (v. 8689), tandis que ce dernier chante des poèmes. Les deux séquences sont présentées comme des rêveries idéales (Lycidas emploie l'irréel du passé) autour d'attitudes caractéristiques. L'activité de chevrier apparaît comme un équivalent du topos du banquet. L'audition de chants poétiques, présente à chaque fois, est l'élément privilégié et dirige l'attention du lecteur sur le rôle joué par la poésie. Au vers 87, Lycidas applique l'adjectif $\kappa \alpha \lambda \grave{\alpha} \varsigma$ aux chèvres de Comatas, juste avant d'ajouter

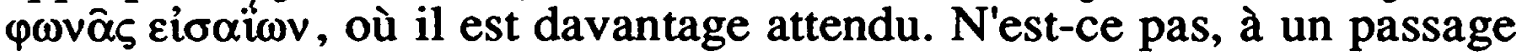
stratégique, faire état d'un particularisme d'homme du métier et d'une naïveté rustique trop accentués ${ }^{24}$ ? Dans le reste du recueil, la règle veut que chevriers et pâtres délaissent leurs troupeaux pour la poésie. Plus qu'elle ne prouve l'attachement de Lycidas à son troupeau ${ }^{25}$, cette notation montre qu'il force le trait, qu'il ironise lui-même sur son état supposé de chevrier. Enfin, si Lycidas prétend avoir composé dans la montagne (v. 51), Simichidas, quoique citadin, assure la même chose avant d'entamer sa propre chanson (v. 92). Il s'agit manifestement d'une formule rhétorique dont l'emploi, tour à tour, par des personnages censés incarner des figures différentes, révèle le caractère arbitraire et interchangeable.

L'idylle 7 est écrite à la première personne du singulier; son récit est assumé par Simichidas. Cependant, sa structure narrative ne permet à aucun moment d'identifier vraiment le personnage de Simichidas. Si l'on compare l'usage qui est fait de la première personne dans le recueil des Idylles et dans les Thalysies, on ne peut manquer d'être sensible au caractère exceptionnel et ambigu qu'elle revêt dans ce texte. Les Idylles se composent soit de poèmes au style direct, qui se présentent comme

23 Voir E. L. BowIE, Early Greek elegy, symposium and public festival, dans J.H.S., 106 (1986), p. 13-35 ; M. VetTA, Poesia e simposio nella Grecia antica, Roma, 1983 et O. MURRAY (éd.), Sympotica, Oxford, 1984.

24 On trouve semblables insistances dans la Pastorale de Longus, où sont faites des allusions précises à la poésie bucolique (par exemple, Chloé I, 10 oublie ses brebis tandis qu'elle tresse une cage pour prendre des sauterelles, comme l'enfant représenté sur la coupe de l'idylle 2, v. 52-54 de Théocrite oublie son déjeuner et ce qui l'entoure). Ainsi Daphnis soigne la beauté de ses chèvres en faisant reluire leurs cornes (IV, 4), veut les mener à l'abreuvoir après avoir été reconnu par son père (IV, 5) et les associe au banquet nuptial, au désagrément des gens de la ville (IV, 38), dans des passages où l'auteur tient à souligner la permanence de sa naïveté et ironise sur le caractère bucolique exemplaire de son personnage.

25 Pour cette lecture, voir G. Giangrande, op. cit., p. 531. 
des dialogues ou des monologues ${ }^{26}$, soit de narrations ${ }^{27}$. Dans ces dernières, le «je» du narrateur ne s'identifie jamais avec celui des discours au style direct qu'il peut introduire dans son récit ${ }^{28}$. L'idylle 7 , où Simichidas est à la fois narrateur de l'idylle, protagoniste des événements qu'il raconte et auteur de certains des discours au style direct et à la première personne qu'il insère dans sa propre narration ${ }^{29}$, présente une configuration narrative tout à fait exceptionnelle. Simichidas appartient à la fois au monde d'où l'on raconte et à celui que l'on raconte. Les avantages et les mystères réservés à un auteur omniscient lui permettent de passer sans cesse de l'un à l'autre, d'interdire toute identification catégorique. Cette ambiguitté est redoublée par la multiplication des points de vue que l'idylle donne sur le personnage de Simichidas : il est successivement connu par sa façon de raconter, par ses propres discours qu'il introduit au style direct, par les descriptions de Lycidas auxquelles il donne lieu. Le lecteur est invité à tirer ses propres conclusions de la confrontation de ces différentes perspectives. Ecart et ambivalence marquent indissolublement Simichidas.

Au cours de l'idylle, Simichidas et Lycidas jouent d'images poétiques, expriment des considérations d'ordre littéraire, chantent des poèmes : ils sont avant tout des poètes. Ils endossent un temps les costumes que leur prête la poésie bucolique pour dénoncer une interprétation littérale de ses figures et de ses caractéristiques, dont ils dévoilent le grotesque et l'invraisemblance en se livrant à une mascarade parodique. Simichidas souligne lui-même qu'il est dangereux d'interpréter à un premier niveau, de façon concrète, les qualifications littéraires et le personnel de la poésie bucolique. Il déclare sans ambiguiité résumer et interpréter des propos qu'il a entendu tenir sur Lycidas :

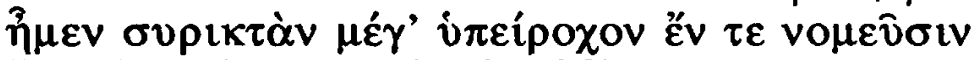

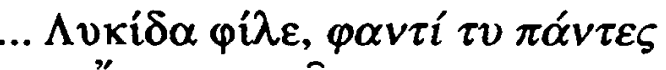

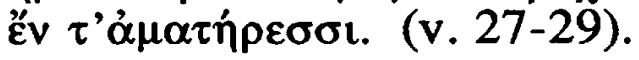

L'emploi conjoint, dans ces vers, de termes tels que musicien ( $\sigma v \rho \imath \kappa \tau \dot{\alpha} v$, v. 28), pâtres (

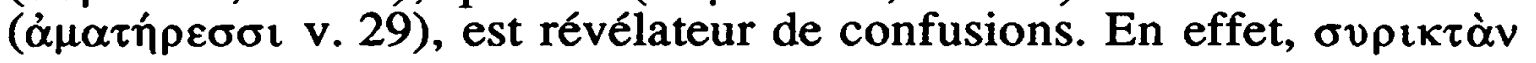

26 Idylles, 1 Thyrsis, 2 Les Magiciennes, 3 La visite galante, 4 Les pâtres, 5 Chevrier et berger, 6 Les chanteurs bucoliques, 10 Les ouvriers ou les moissonneurs, 12 Le bien-aimé, 14 L'amour de Kynisca, 15 Les Syracusaines, 16 Les Charites, 17 Eloge de Ptolémée, 22 Les Dioscures, 28 La quenouille, 29 L'enfant aimé (I), 30 L'enfant aimé (2).

27 Idylles, 7 Les Thalysies, 11 Le Cyclope, 13 Hylas, 18 Epithalame d'Hélène, 24 Héraclès enfant.

28 Idylles, 7 Les Thalysies, 11 Le Cyclope, 18 Épithalame d'Hélène, 24 Héraclès enfant.

$29^{\circ}$ V. $27-41$ et $91-127$ 
désigne un joueur de syrinx, instrument de musique par excellence du

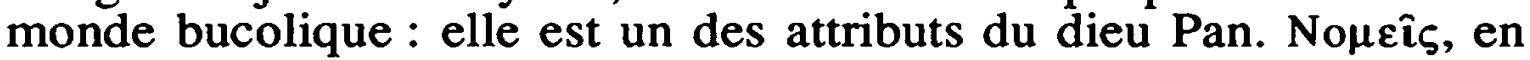
revanche, n'apparaît que dans le titre, d'origine incertaine, de l'idylle 4. Celle-ci se compose, de toutes façons, d'un entretien familier et sans art apparent, qui ne désigne pas ses protagonistes comme des artistes. Les trois autres occurrences du terme se trouvent dans des idylles dont l'attribution à Théocrite est contestée, les idylles 9, les Chanteurs bucoliques (v. 29), et 25, Héraclès tueur de lion (v. 109 et 122). Nouعîs qualifie Daphnis et Ménalcas dans l'idylle 9, mais le texte semble être une variation à partir de l'idylle 7 , ce qui rend difficile son exploitation. En revanche, il n'y a aucun sous-entendu poétique possible à l'emploi de voukîs dans l'idylle 25 , où il s'applique à une catégorie de paysans, comme dans les pièces de Théocrite lui-même. Les voukî̧ ne constituent pas un groupe de référence poétique. Quant aux moissonneurs, ils ne sont jamais mentionnés ailleurs dans les Idylles, que dans le titre de l'idylle 10 , dont les protagonistes sont de simples paysans. Ils n'incarnent donc pas non plus de valeur poétique. Pour signifier le talent de Lycidas, Simichidas l'assimile à des personnages qui ne sont pas des chanteurs. La poésie bucolique ${ }^{30}$ tient son sens d'une construction arbitraire, qui est à elle-même son propre système de référence. Une transposition littérale, dans l'ordre du concret, de ses catégories, détruit sa logique et rend son propos absurde.

La comédie du travestissement des personnages incite donc le lecteur de l'idylle 7 à considérer toujours l'écart et la part de convention inhérente à ces représentations. Simichidas et Lycidas le mettent en garde contre les interprétations immédiates, non distanciées, de la poésie bucolique, qui lui font perdre tout son sel.

La connivence dont font preuve Lycidas et Simichidas invite à relire le passage où Lycidas donne son bâton à Simichidas (v. 37-121). En effet, il ne s'agit plus, dès lors que Simichidas et Lycidas agissent de concert, d'une simple consécration d'un des deux protagonistes. Il faut déchiffrer la raison et le sens de la scène que jouent les personnages.

Le don du bâton est provoqué par une déclaration de Simichidas :

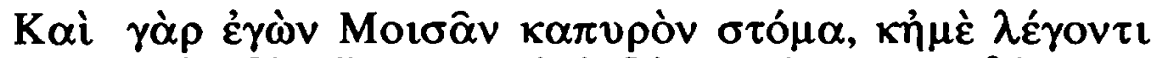

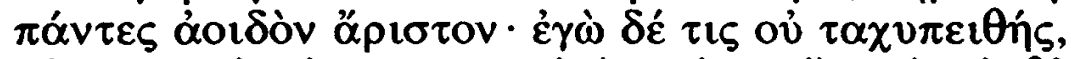

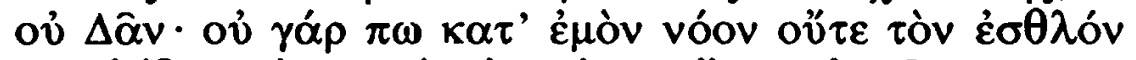

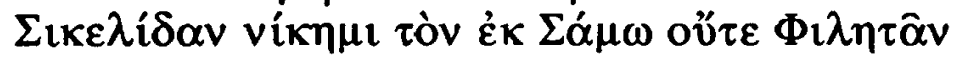

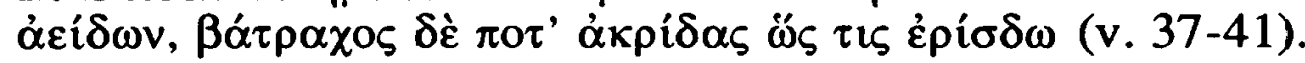

30 Le même procédé est employé dans l'idylle 11. Polyphème évoque la beauté de Galatée en utilisant la forme traditionnelle de l'éloge, mais il recourt dans ses comparaisons à des référents empruntés à son expérience directe qui tranchent et détonnent dans le contexte rhétorique qu'il n'a pas compris ou identifié comme arbitraire. 
La plupart des commentateurs tentent de décrypter dans ces vers, à travers un travail d'identification complexe, une appréciation de la littérature contemporaine par Théocrite. Si l'on effectue d'abord une lecture littérale de ce passage, il signifie que Simichidas met en doute la qualité de ses créations par rapport à celles de Sikélidas et Philétas. C'est la comparaison avec Sikélidas et Philétas qui a le plus d'intérêt : elle exprime l'idée que le poète crée par rapport à d'autres, par

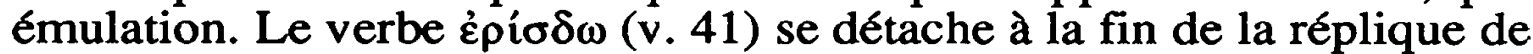
Simichidas. Il est employé sans ambiguïté dans l'idylle 5 (v. 67-136) pour caractériser l'exercice auquel se livrent des chanteurs quand un prix est en jeu. Cette vision est faite pour plaire à Lycidas : l'adverbe $\dot{\varepsilon} \pi i \tau \alpha \delta \varepsilon \varsigma$ (v. 42) stipule que Simichidas agit par intention et qu'il connaît les options esthétiques de son interlocuteur.

Le don de son bâton par Lycidas à Simichidas illustre cet accord. Il en précise les termes. La scène est une récriture ironique des vers 22 à 31 de la Théogonie d'Hésiode. En effet, Lycidas, qui apparaît comme une caricature de chevrier, joue, dans l'idylle 7, le rôle de la Muse chez Hésiode. Or elle n'avait pas de mots assez durs pour mépriser les

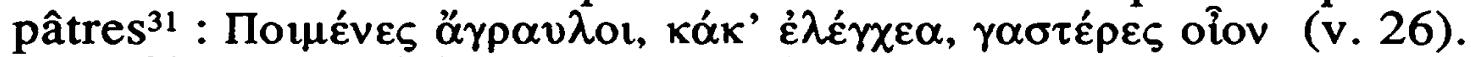

Si l'on considère le texte de l'idylle 7 de plus près, on s'aperçoit qu'il ne procède pas seulement à une inversion du modèle fourni par la Théogonie, mais fait écho à une nouvelle conception de la poésie. Lycidas donne son bâton à Simichidas : la source de la poésie n'est plus la Muse, une divinité, mais un être humain et un poète. En remplaçant la Muse par un chevrier, proche parent du berger Hésiode lui-mểme ${ }^{32}$, Théocrite n'invite-t-il pas, dans un système d'inspiration où la littérarité tient une place primordiale, à tenir l'Hésiode représentant du monde rural, l'Hésiode des Travaux et des Jours, pour le modèle originel de la poésie bucolique ? Comme dans le discours précédent de Simichidas, est affirmée l'idée que la poésie consiste en emprunts, de poète à poète, d'œuvre à œuvre. D'autre part, Lycidas explique qu'il donne son bâton

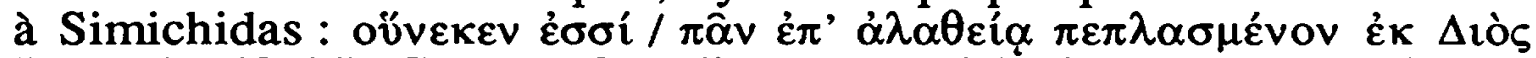
Épvos (v. 43-44). Ces vers font directement écho à une proclamation de

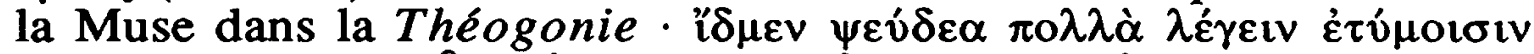

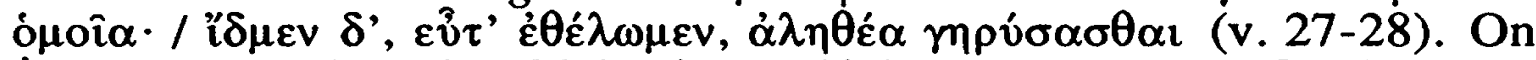
observe cependant chez Théocrite un déplacement du sens. Ce n'est plus l'émetteur, c'est-à-dire la Muse, qui est porteur du discours vrai, mais le récepteur, Simichidas. La vision de la création poétique proposée par Lycidas est différente de celle de la poésie archaïque, où la Muse représente le poids de la tradition orale. Désormais, l'accent est mis sur

31 Voì̀ aussi G. GIANGRANDE, op. cit., p. 522, n. 72.

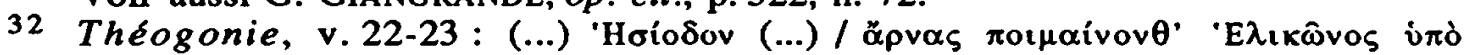
$\zeta \alpha \theta$ toro. 
l'action du poète lui-même. Comme Simichidas est apparu jusque-là dans l'idylle sous le jour de dissonances et de contradictions, Lycidas suggère peut-être même la place essentielle que la distance consciente et l'art de la variation doivent occuper dans cette nouvelle poétique.

En parlant des Motoồv ǒpvixєs (v. 47), Lycidas évoque l'attitude à l'égard de la création poétique qu'il juge néfaste. Comparé à ce que révèle le comportement de Simichidas ou à la réorientation de la scène de la Théogonie à laquelle se livre Lycidas, ce pôle négatif permet de

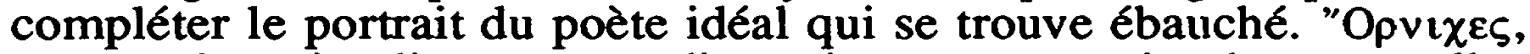
«les poules», implique un son disgracieux, au contraire des sauterelles du vers 41 . Lycidas désigne de manière satirique les mauvais poètes. Ce sont ceux qui veulent élever une maison aussi haute qu'une montagne. Orgueil et sublime sont dénoncés, avec un vocabulaire ( $\tau \dot{\varepsilon} \kappa \tau \omega v, v .45)$

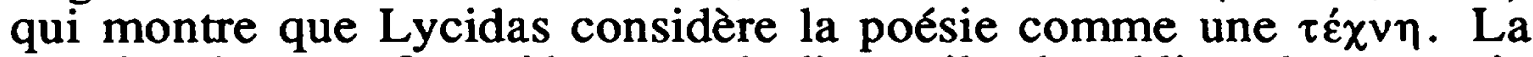
mention du mont Oromédon rattache l'orgueil et le sublime des mauvais poètes à la conception traditionnelle de la poésie inspirée, puisqu'il existe un culte d'Apollon Oromédon ${ }^{33}$. A la fin de la joute poétique de Lycidas et Simichidas, le lecteur apprend que Lycidas prend le chemin de Pyxa: E. L. Bowie signale une scholie qui mentionne un culte d'Apollon Oromédon à Pyxa ${ }^{34}$. C'est signifier, en accordant à Lycidas de se rendre où les autres poètes souhaitent aller, que Simichidas et Lycidas savent, eux, quelle poésie il faut écrire. Lycidas évoque alors Homère : Xîov áotò̀v (v. 47-48). Cette caractérisation par l'origine géographique du poète était déjà employée par Simichidas pour

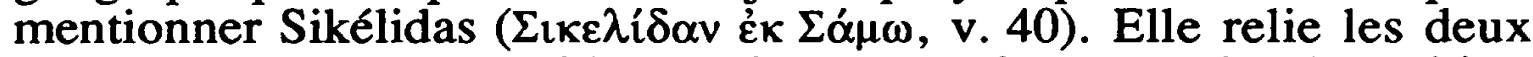
discours. Les vers de Lycidas expriment peut-être une prise de position dans la querelle que suscite à l'époque hellénistique la question de l'écriture épique ${ }^{35}$. Ils généralisent surtout l'idée que vient d'exprimer Simichidas : il est vain de prétendre égaler un modèle prestigieux. Dans les propos de Simichidas, cette constatation s'applique quasiment à des contemporains, Sikélidas et Philétas. En évoquant désormais Homère, le poète par excellence, Lycidas transforme en règle générale la remarque de son interlocuteur. Simichidas et Lycidas signifient que se réclamer de la tradition de l'inspiration ou de la tradition poétique ne suffit pas pour accéder à la création véritable et au talent. Le verbe

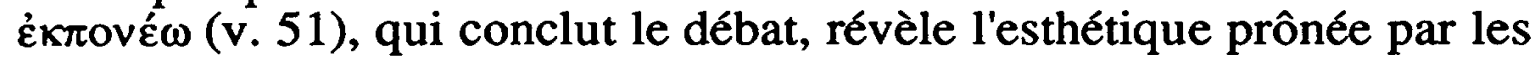

33 Voir E. L. BowIE, op. cit., p. 70-71.

34 Op. cit., p. 70-71.

35 On a longtemps pensé que ces vers désignaient la volonté de continuer à écrire des épopées de type homérique, et en particulier Apollonios de Rhodes, auquel une querelle aurait opposé Théocrite. Ils sont alors interprétés comme une approbation de l'esthétique de Callimaque contre celle d'Apollonios. Plus récemment, la désignation d'Apollonios a été remise en cause au profit d'une accusation plus générale des auteurs d'épopées hellénistiques que nous ne possédons plus. 
protagonistes de l'idylle : il désigne un travail personnel minutieux. À la fin de son intervention, Lycidas revient sur l'un des premiers thèmes mentionnés par Simichidas, la modestie du poète face à sa tâche. De manière symbolique, la montagne à laquelle le poète critiqué par Lycidas veut égaler son œuvre (v. 46), devient pour Lycidas et Simichidas un lieu réel, où ils prétendent avoir composé leurs propres poèmes (v. 51 et 92). Dans ce cas, refuge des pauvres bergers, elle symbolise l'humilité à laquelle Lycidas et Simichidas veulent s'en tenir, alors même qu'ils sont clairement désignés comme des poètes avertis dans le reste du texte.

Après que Lycidas a récité son poème, Simichidas déclare :

$\Lambda v \kappa i ́ \delta \alpha \varphi \dot{\imath} \lambda \varepsilon, \pi \circ \lambda \lambda \dot{\alpha} \mu \varepsilon^{\prime} v \not ̈ \alpha \lambda \alpha$

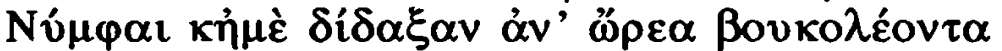

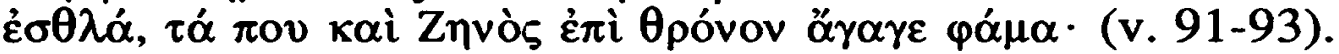

Ces vers demandent à être examinés de façon détaillée, car, dans un premier temps, ils évoquent si fidèlement le texte de la Théogonie, qu'on peut être tenté de croire que Simichidas se rattache en réalité à la tradition, rétablit le rôle des Muses et celui de l'inspiration dans la création poétique. Hésiode écrit en effet au début de la Théogonie :

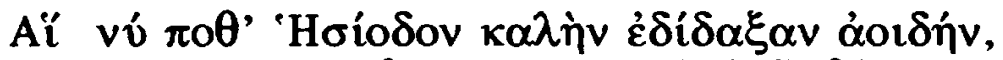

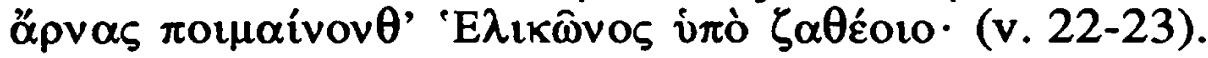

Ensuite, les Muses offrent leur houlette à Hésiode et ce dernier

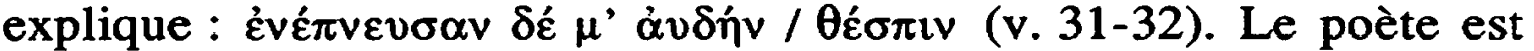
l'organe des Muses. On se souvient que Simichidas déclare aussi être :

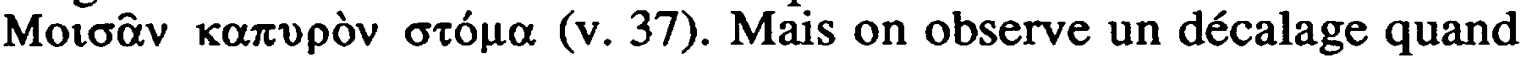
Simichidas dit que la renommée a porté ses chants jusqu'au trône de

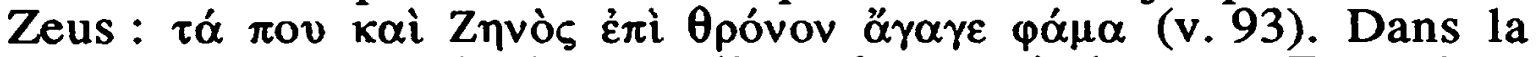
Théogonie, ce sont les Muses elles-mêmes qui charment Zeus, dans $l^{\prime} O 1 y m p e^{36}$. Le poète ne fait que répéter leurs paroles. De façon significative, Hésiode conjure les Muses de lui raconter la genèse des dieux, puis la Théogonie commence véritablement, sans qu'il soit

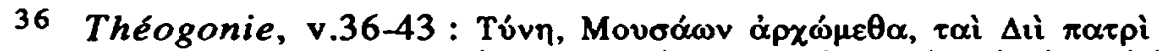

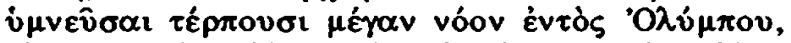

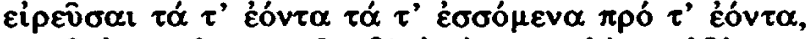

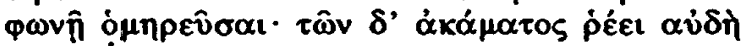

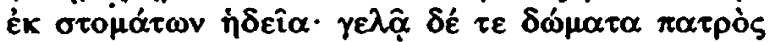

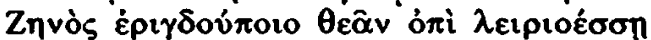

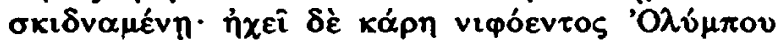
$\delta$ óp $\alpha \tau \alpha^{\prime} \tau^{\prime} \dot{\alpha} \theta \alpha v \alpha \dot{\alpha} \tau \omega v$. 
possible de déterminer s'il s'agit du discours des Muses ou de celui d'Hésiode :

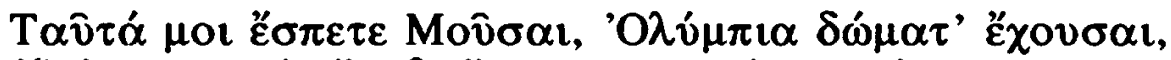

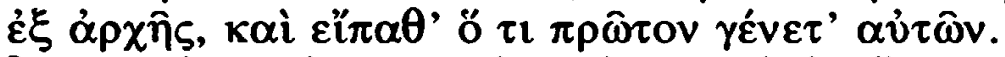

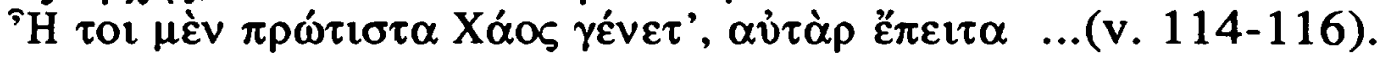

Au contraire, chez Théocrite, de nombreuses expressions reviennent sur

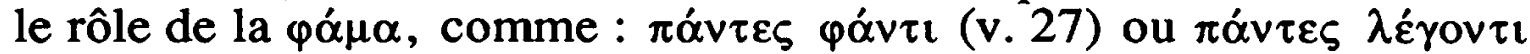
(v. 37). L'immédiateté, la rigoureuse équivalence entre la parole des Muses et celle de l'homme, qui existent dans la Théogonie, ont disparu. Désormais, l'importance du milieu littéraire est décisive. Le sens du passage que Simichidas emprunte à la Théogonie est altéré. Le rappel de ces vers ne signifie pas un retour à la poétique qu'ils expriment. Il convient de s'interroger sur la valeur de l'adverbe кaí, qu'utilise Simichidas au vers $92:$ Nó $\mu \varphi \alpha \imath \kappa \grave{\eta} \mu \grave{\varepsilon} \delta i \delta \alpha \xi \alpha v$. Il peut constituer une allusion à Hésiode. Il peut aussi faire référence à Lycidas. Simichidas déclare d'ailleurs ensuite que Lycidas est «l'ami des Muses» ( $\varphi$ í $\lambda_{\circ}$

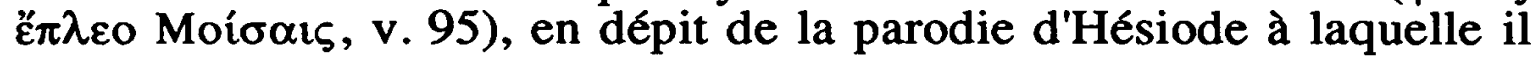
s'est livré. On a déjà remarqué qu'après avoir échangé leurs poèmes Lycidas et Simichidas se séparent, et que Lycidas prend le chemin d'un sanctuaire d'Apollon Oromédon. Lycidas et Simichidas ne refusent pas l'existence ou le patronage des Muses, mais en redéfinissent les modalités. Simichidas, en citant presque mot à mot le texte d'Hésiode, revendique l'héritage de la tradition et sa garantie pour la poétique que Lycidas et lui tirent de la redistribution des rôles à laquelle ils procèdent. Il s'efforce de faire apparaître leur méthode comme la seule valable et

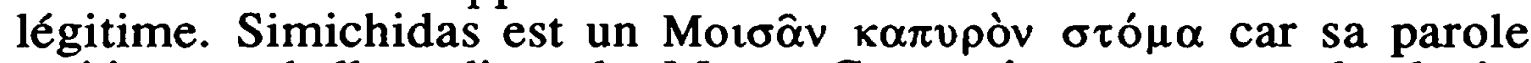
poétique est belle et digne des Muses. Ceux qui se contentent de plagiat sont des Moıoâv őpvixєs. Le mérite du poète auprès des Muses ne dépend pas de l'adéquation de son discours avec une parole qu'elles insufflent en lui, mais de la qualité de l'œuvre qu'il produit. Cette révision explique que Lycidas puisse donner définitivement son bâton à Simichidas ék Moıoồ (v. 129) : un nouveau pacte a été conclu avec ces dernières.

Une dernière vérification de la complicité de Lycidas et Simichidas est offerte au lecteur, lorsque le don du bâton est évoqué une seconde fois, après l'échange des poèmes de Lycidas et Simichidas. Dans la Théogonie, le don du bâton précède le chant d'Hésiode, dont il est la condition nécessaire et un indispensable préalable. Dans l'idylle 7, d'abord annoncé avant l'échange des poèmes, rendu effectif après, il consacre l'accord des deux poètes. Le choix du vocabulaire qui sert à désigner le bâton que Lycidas remet à Simichidas montre leur volonté de dégonfler l'héroïsme et la grandiloquence d'une conception de la poésie 
dictée par les dieux, au profit de la littérarité et du labeur conscient. La Muse, dans la Théogonie, offre à Hésiode un okñ $\pi \tau$ pov (v. 30), qui a une forte coloration épique et désigne chez Homère le bâton que tient le roi en signe de son autorité ou l'orateur à l'assemblée ${ }^{37}$. Lycidas, quant à lui, préfère parler de kopv́va (v. 43), qui désigne une massue, un gourdin, une houlette de berger, sans connotation spécifique. C'est une première réduction. Lors de la seconde mention du don du bâton, vers 127 , il est appelé $\lambda \alpha \gamma \omega \beta$ ó $\lambda$ ov par Simichidas, faisant alors allusion à un «bâton de berger crochu avec lequel on peut attraper un lièvre» ${ }^{38}$, ce qui achève d'orienter vers la parodie et la franche comédie la réécriture de la scène d'Hésiode. En revanche, l'adjectif $\xi_{\varepsilon ı v}$ íiov, dans les mêmes vers de Simichidas, porte la connotation épique ôtée au bâton :

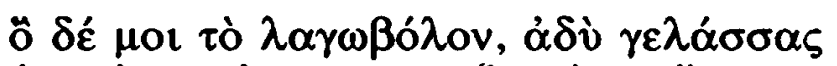

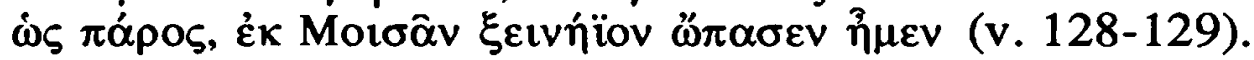

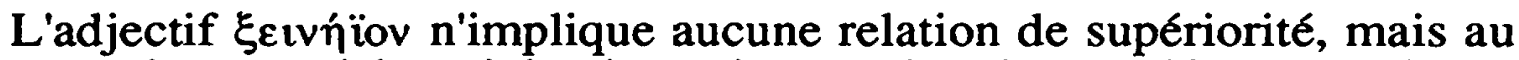
contraire une réciprocité qui suggère que la scène se déroule de l'aveu des deux personnages et selon un schéma qu'ils ratifient tous deux. Ce terme déplace définitivement l'accent du texte d'Hésiode. Ce n'est plus le présent des Muses qui est mis en valeur, mais la connivence des deux poètes.

L'évolution des termes employés pour désigner le bâton ne marque pas seulement une baisse de prestige. Le $\lambda \alpha \gamma \omega \beta$ ó $\lambda$ ov est, au même titre que la syrinx, un attribut caractéristique de $\operatorname{Pan}^{39}$, dieu des pâtres et des bergers. On peut se demander si la nouvelle poétique que formulent Lycidas et Simichidas n'entraîne pas la modification du patronage que les Muses exerçaient seules jusque-là.

La présence de Pan anime en réalité tout le poème. Depuis le début des Thalysies, le décor dressé par les deux poètes laisse prévoir une telle intrusion. La scène se passe en plein midi : il s'agit de l'heure où se produisent la plupart des apparitions divines, mais elle est particulièrement significative dans la mythologie panique ${ }^{40}$, et Théocrite montre qu'il en a connaissance dans l'idylle 1 (v. 15-16) : le chevrier refuse de

37 Voir E. BENVENISTE, Le vocabulaire des institutions indo-européennes, II : Pouvoir, droit, religion, chap. 3 La royauté hellénique, p. 29-32.

38 P. Chantraine, op. cit. Ce renchérissement, venant de la part de Simichidas, ne peut être fortuit et est bien la preuve que ce personnage n'est pas seulement l'individu naïf décrit en particulier par G. GIANGRANDE, op. cit.

39 Voir P. BoRgeaud, Recherches sur le dieu Pan, Genève, 1979 (Bibliotheca Helvetica Romana, 17), p. 83 et p. 101.

40 Voir P. BORGEAUD, op. cit., p. 93 et p. 167-168. 
jouer de la musique à midi pour ne pas réveiller Pan ${ }^{41}$. Le paysage aride évoqué n'est pas sans ressemblance avec celui de l'Arcadie, terre natale du dieu ${ }^{42}$. Si Lycidas porte une peau de bouc, c'est probablement par allusion à Pan lui-même. La mention des Nymphes, v. 92, au lieu des Muses, poursuit la tendance amorcée. Puis, l'échange des poèmes entre Lycidas et Simichidas figure un équivalent exact des nombreuses joutes bucoliques ${ }^{43}$ représentées dans les Idylles. Mais force est alors de constater plusieurs contradictions qui remettent en cause l'idée d'un remplacement pur et simple de la conception poétique que symbolisent les Muses par celle de Pan. Lycidas et Simichidas, contrairement au chevrier de l'idylle 1 , transgressent la convention selon laquelle midi doit être l'heure du silence. Cet affranchissement des poètes est renforcé par le fait qu'ils sont explicitement désignés, au début du poème, comme les seules créatures éveillées parmi des corneilles et des lézards assoupis. Le bruit que Simichidas fait en heurtant les pierres (v. 25-26) prend alors un relief accru : il représente une menace pour le sommeil du dieu. L'ambivalence du texte renvoie, pour son élucidation, aux poèmes de Lycidas et Simichidas, sertis au centre de l'idylle, dans un mouvement qui les met en valeur.

Le contenu des poèmes qu'échangent Lycidas et Simichidas manifeste la même complémentarité que les propos qui les entourent, dont ils reprennent et enrichissent chacun des thèmes. Un court résumé suffit ainsi à révéler les tensions qui animent les deux passages. La chanson de Lycidas (v. 52-89) a les apparences d'un $\pi \rho \circ \pi \varepsilon \mu \pi \tau \iota \kappa o ́ v$, poème composé à l'occasion du début d'un voyage ${ }^{44}$. Pourtant, si elle sacrifie au thème récurrent du souhait d'une bonne traversée ${ }^{45}$, elle n'accorde aucun développement au lieu commun des dangers de la

41 Le chevrier refuse de chanter, car il ne faut pas réveiller Pan dans son sommeil.

Voir P. Borgeaud, op. cit., p. 167, n. 126.

42 Voir P. BorgeaUd, op. cit., p. 94.

43 Voir, chez Théocrite, les idylles 1 Thyrsis, 5 Chevrier et berger, 6 Les chanteurs bucoliques, 10 Les Moissonneurs.

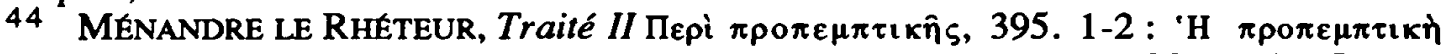

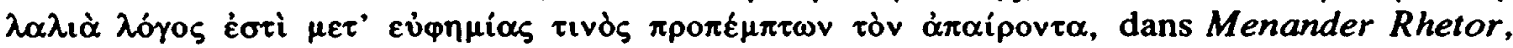
edition, translation and commentary by D. A. RuSSEL, N. G. WILsON, Oxford, Clarendon Press, 1981.

45 F. CaIRns, Generic composition in Greek and Roman Poetry, Edinburgh, Edinburgh University Press, 1972 , p. $115: \ll(\ldots)$ we may categorize some of the material of these propemptika as follows: (i) good wishes and divine help for outlined journey ending at destination; (ii) description of destination; (iii) speaker's wish to accompany / excuse for not doing so; (iv) reiteration of good wishes and divine help». 
navigation ${ }^{46}$, qui tient une place de choix dans les autres exemples de

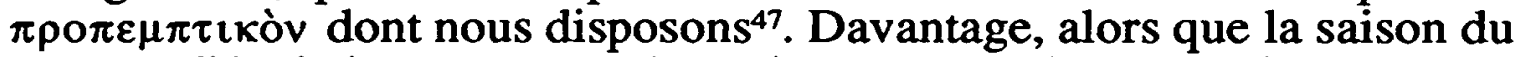
voyage d'Agénéax correspond au pire moment du mare clausum ${ }^{48}$, le narrateur ne manifeste aucune inquiétude pour le sort du voyageur. De même, l'attention qui doit être dévolue à ce dernier jusqu'à la fin du poème, dont il s'avère le sujet principal ${ }^{49}$, est suspendue au profit de l'évocation du narrateur sédentaire : Agéanax n'est plus mentionné après la ligne 69 , soit pendant les deux tiers du poème. La chanson de Lycidas, qui fait référence à un type de poème précis, contrevient cependant à sa construction et à son contenu ordinaires. Le poème de Simichidas n'est pas moins curieux. S'il s'ouvre sur l'évocation des amours du poète avec Myrto (v. 96-97), il change ensuite totalement de sujet et évoque celles d'Aratos pour un jeune homme. Simichidas demande à Pan de favoriser Aratos, mais d'une façon irrévérencieuse et naïve. Le poème adopte alors la forme d'un $\pi \alpha \rho \alpha \kappa \lambda \alpha v \sigma i \theta v \rho o v$, où

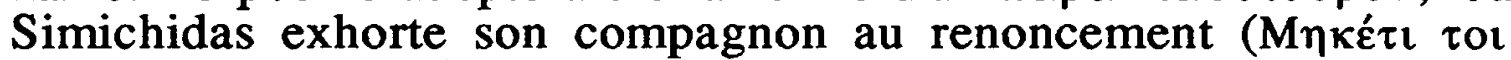

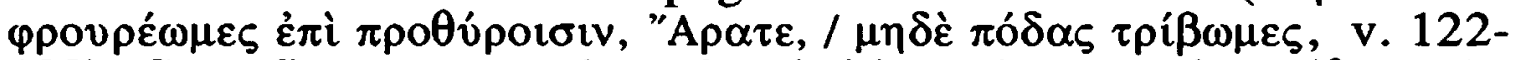
$123)$. Cette fin trangresse le code générique du $\pi \alpha \rho \alpha \kappa \lambda \alpha v \sigma i ́ \theta v \rho v^{50}$, où, quelle que soit la déception, la résignation, la révolte de l'amant, il demeure sous la loi de celui ou celle qu'il aime ${ }^{51}$. D'autre part, forme

46 Sur l'importance de ce topos dans les $\pi \rho \circ \varepsilon \varepsilon \mu \tau \iota$ кó, voir F. CAIRNS, op. cit., p. 52 et R. G. M. Nisbet, M. HubBaRD, Commentary on Horace's Odes I, Oxford, Clarendon Press, 1970, p. 40-42.

47 Voir les références données dans Menander Rhetor, op, cit., p. 304-305 et F. CAIRNS, op. cit., p. 284-285.

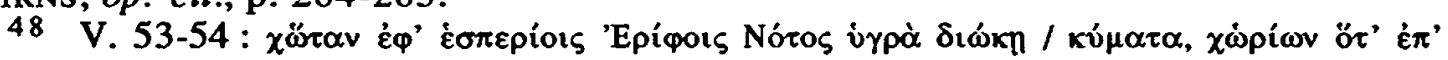

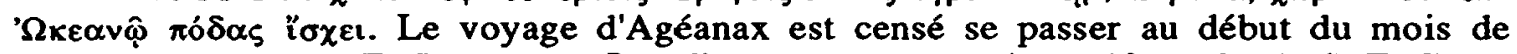
novembre. Voir Ph.-E. LEGRAND, Bucoliques grecs, op. cit., p. 10, n. 6 ; A. S. F. Gow, Theocritus, edited with a commentary, II (Cambridge, Cambridge University Press, 1950), p. 145-146 ; Theocritus, Select Poems, edited with an introduction and commentary by K. J. DOVER, London, MacMillan, 1971, p. 155-156.

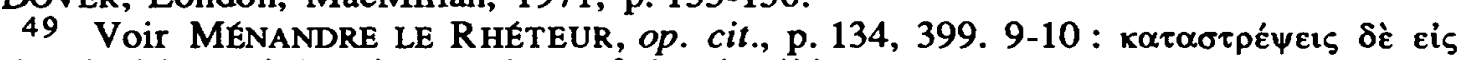

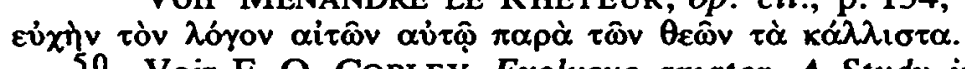

50 Voir F. O. Copley, Exclusus amator. A Study in Latin Love Poetry, 1956 (Am. Phil. Ass., 17), The Greek Paraclausithyron. General characteristics, p. 1-27.

51 F. O. COPLEY, op. cit., p. 1: « [The lover] sings his song, in which he may combine a plea that the girl will yet relent, a warning of the lonely days to come, when she will be too old for love, a protestation against her cruelty, and a picture of how his own sufferings. Sometimes he threatens suicide. In the end he may hang his garland on her door, or throw it at the threshold; sometimes he scribbles on the door some scraps of verse. Then he lies down in the doorway, there to remain until morning». Voir aussi S. L. TARAN, The Art of Variation in the Hellenistic Epigram, Leiden, E. J. Brill, 1979, Chapter

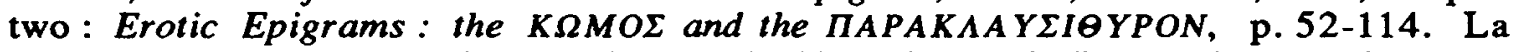
constance des amants est mise en valeur par le thème de la nuit d'orage, du mauvais temps, etc... On trouve un écho de cette insistance dans l'idylle 3 de Théocrite (v. 52-55), où le narrateur menace de se laisser dévorer par les loups sur le seuil d'Amaryllis. 
littéraire urbaine s'il en est ${ }^{52}$, le $\pi \alpha \rho \alpha \kappa \lambda \alpha v \sigma i ́ \theta v \rho o v$ implique un contraste entre la situation dans laquelle Simichidas prétend avoir composé son poème, c'est-à-dire en gardant ses vaches dans la montagne (v. 92) $)^{53}$, et la manière dont il se figure à l'intérieur même de son poème : il accompagne Aratos (v. 122-127, écrits à la deuxième personne du pluriel) devant la porte de Philinos. La forme du $\pi \alpha \rho \alpha \kappa \lambda \alpha v \sigma i \theta v \rho o v$ est elle-même pervertie: l'usage veut que le $\pi \alpha \rho \alpha \kappa \lambda \alpha v \sigma i \theta v \rho o v$ suive un banquet, où les jeunes gens ont beaucoup bu, et un $\kappa \hat{\omega} \mu \varsigma^{54}$. Or, dans le poème de Simichidas, l'invitation à l'ó $\sigma v \chi i ́ \alpha$ et au banquet vient après une première station devant la porte de Philinos et constitue la récompense du renoncement (v. 126). Simichidas, comme Lycidas, invertit les termes du genre auquel il semble d'abord se référer.

Les poèmes de Lycidas et Simichidas doivent donc être placés en parallèle et étudiés l'un par rapport à l'autre. D'ailleurs, le poème de Lycidas s'achevant sur le nom de Comatas (v. 89), l'envoi de celui de Simichidas se compose du nom de Lycidas (v.91) et effectue une liaison stylistique explicite avec le précédent. Les relations amoureuses évoquées dans les deux textes sont encore un indice des relations étroites qu'ils entretiennent. Au-delà d'un simple phénomène d'échos, elles les constituent en une totalité signifiante.

En effet, on observe que :

- dans le poème de Lycidas : (1) Lycidas aime Ageanax

(2) Daphnis aime Xenéa,

- dans celui de Simichidas : (3) Simichidas aime Myrto

(4) Aratos aime Philinos.

A chaque fois, se trouvent mis en scène un couple homosexuel et un couple hétérosexuel, mais la tonalité de leurs relations est inversée d'un texte à l'autre. Ainsi la chanson de Lycidas présente :

(1) l'amour heureux d'un homme pour un autre homme

(2) l'amour malheureux d'un homme pour une femme,

et celle de Simichidas: (3) l'amour heureux d'un homme pour une femme

(4) l'amour malheureux d'un homme pour un autre homme.

52 Une des principales ressources humoristiques de l'idylle 3 consiste précisément à situer cette scène à la campagne, puisque le narrateur quitte ses chèvrès au début du poème pour se rendre devant la «caverne» (v. 6) d'Amaryllis.

53 Sur l'invraisemblance de cette prétention, voir selon une perspective différente $G$. GiANGRANDE, op. cit., p. 508-511.

54 Voir F. O. Copley, op. cit., p. 4-5. 
La symétrie de cette construction doit convaincre que les deux poèmes constituent un tout, en dépit de leurs divergences de surface. Ils résument l'ensemble des situations amoureuses possibles, du moins d'un point de vue masculin, qui compte seul. On note d'autre part qu'ils ont en commun la particularité de cesser rapidement de s'occuper de la situation amoureuse qui suppose un certain équilibre (1 et 3 ) et inclut le narrateur.

Il est possible de remarquer ensuite que les cas (2) et (4) supposent l'existence d'un troisième personnage, un poète (Tityre et Aristis), dont le rôle est apparemment de chanter l'amour malheureux du personnage évoqué. «Apparemment», car, dans le poème de Lycidas, Tityre est censé chanter de façon explicite (v. 72, le futur est employé :

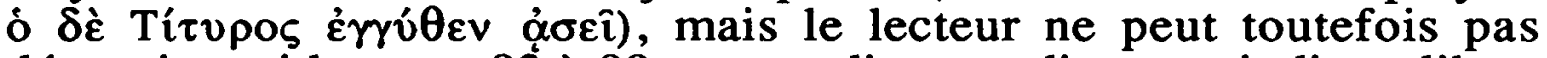
déterminer si les vers 83 à 89 sont au discours direct ou indirect libre, s'ils sont une citation du poème de Tityre par Lycidas, un souvenir, une récriture ou aucune autre forme émanant de Lycidas lui-même. Dans le poème de Simichidas, Aristis, quoique averti de la passion d'Aratos et excellent poète (v. 99-102), ne se voit attribuer aucun chant particulier, tandis que la suite du texte et la célébration des amours d'Aratos sont clairement prises en compte par Simichidas lui-même. Tityre et Aristis semblent avoir pour fonction exclusive de montrer que le poème est toujours délivré par un personnage extérieur ${ }^{55}$. Ils servent d'écran entre le poète et son sujet. Lycidas et Simichidas illustrent chacun à leur tour le refus qu'un besoin affectif personnel et immédiat soit à l'origine de la création poétique : ils semblent ne faire allusion à leurs amours, aussitôt oubliées, que pour convaincre le lecteur de cette vérité. Simichidas se présente clairement comme l'auteur de la totalité de son poème : Aristis, par sa seule mention, rappelle la situation formulée dans la chanson de Lycidas ; Simichidas peut ainsi aller plus loin et poser, à travers son propre exemple, que le poète, quand bien même il serait partie de l'action qu'il narre, garde une position d'observateur et une distance qu'exprime l'invitation au renoncement (v. 122-127). Ce double mouvement, où interviennent simultanément engagement et distance consciente, s'applique à la représentation de Lycidas et Simichidas dans l'idylle 7 elle-même.

Une étape supplémentaire peut être franchie. Lycidas se figure à partir du vers 65 au comble de la félicité, en dépit de l'absence de son amant : un simple vœu à l'adresse d'Agéanax lui permet de jouir d'un bonheur dont l'élément le plus développé est l'audition des chansons de Tityre. Or, pour conclure, Simichidas invite Aratos à parvenir à un état

55 C'est la structure qu'on retrouve dans les idylles qui se présentent comme un récit a la première personne. Le narrateur n'est jamais le sujet de sa narration ni un acteur des événements qu'il raconte. 
d'ó $\sigma v \chi i ́ \alpha$ (v. 126) qui est précisément celui de Lycidas dans son poème. N'est-ce pas, au terme de sa chanson, dire que la question de la relation amoureuse ne compte pas en elle-même, mais seulement dans la mesure où elle est prétexte à poésie, et qu'elle doit être appréciée en fonction de cette ambition ? Lycidas, dès lors, n'atteint-il pas au bonheur dans l'absence parce qu'elle lui est occasion de poésie ? Les poèmes de Lycidas et Simichidas n'invitent pas seulement le lecteur à se convaincre de la distance du poète par rapport à son sujet, mais ajoutent que le sujet ne vaut que par le poème qu'il permet de créer, le plaisir poétique dont il est l'occasion. L'évocation de Comatas offre une vérification supplémentaire de cette idée. Les aventures de Comatas, quoique pénibles, sont déclarées $\tau \varepsilon \rho \pi v \alpha \grave{\alpha}(v .83)$. Lycidas se réfère à la tradition présente chez Homère et Hésiode ${ }^{56}$, selon laquelle la poésie possède le pouvoir de transformer les peines de l'un en plaisir pour l'autre. On observe toutefois une gradation entre la position d'Hésiode et celle de Théocrite. Chez Théocrite, c'est l'individu même qui a souffert qui parvient au bonheur grâce à la poésie. Comatas est déclaré $\mu \alpha \kappa \alpha ́ \rho \imath \sigma \tau \varepsilon$ (v. 83) ; il a été physiquement sauvé par les abeilles, séduites par sa parole poétique. La faculté de la poésie à créer le bonheur est formulée de manière explicite dans l'idylle 11 :

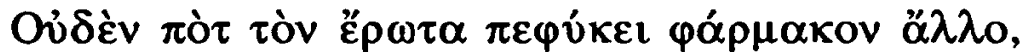

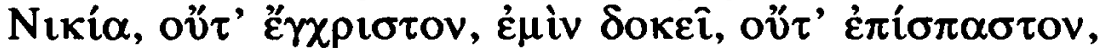
ทे $\tau \alpha \grave{i ̀ ~ \Pi \imath \varepsilon \rho i ́ \delta \varepsilon \zeta ~(v . ~ 1-3) . ~}$

Insister sur l'affranchissement qu'autorise la poésie à travers

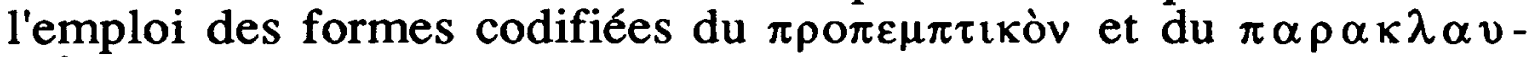
oílupov, c'est-à-dire de poèmes de circonstance ${ }^{57}$, équivaut de la part de l'auteur à un tour de force puisque, de cette façon, il parvient à insister sur la part de convention première et inhérente à la poésie, tandis que les libertés prises avec les «lois» du genre pointent ironiquement sur le libre-arbitre et les ressources qui demeurent à leur utilisateur. Le thème de la félicité du poète vient compléter et exalter cette vision d'un épanouissement poétique.

On retrouve dans les poèmes de Lycidas et Simichidas la question du patronage de la poésie par une divinité symbolique. Les deux poèmes sont contenus à l'intérieur de la séquence du don du bâton de Lycidas,

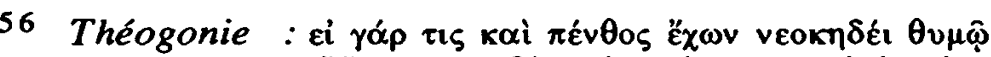

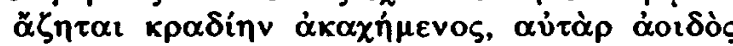

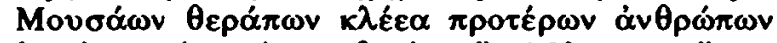

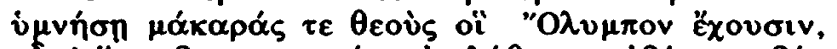

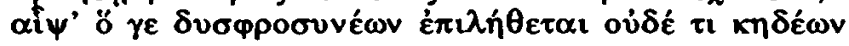

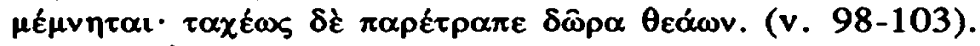

Voir S. L. TARAN, op. cit. 
où l'influence de Pan vient doubler, sinon remplacer, celle des Muses. Or ils ont souvent été opposés : la chanson de Lycidas représenterait un versant apollinien, dominé par l'idée de mesure et d'ó $\sigma v \chi i ́ \alpha$, tandis que celle de Simichidas est directement adressée à Pan, avec une crudité et une irrévérence propres au dieu.

En réalité, Pan habite les deux poèmes. Certes, il n'est pas cité par Lycidas, mais sa chanson, à l'intérieur d'une forme urbaine et érudite, réserve une place considérable à la veine bucolique. Agéanax, au début, fait voile vers Mytilène (v. 52). E. L. Bowie montre que Théocrite choisit vraisemblablement de nommer Mytilène (et d'en faire la patrie de Lycidas et de son amant) par référence à un poème bucolique de Philétas, auquel la campagne autour de Mytilène sert de décor ${ }^{58}$. Ce détail géographique ne peut passer inaperçu aux yeux du lecteur attentif de l'idylle 7, dont la localisation à Cos constitue un premier hommage à Philétas ${ }^{59}$. De plus, les personnages cités sont tous des pâtres et des chevriers, qui appartiennent au personnel ordinaire de la poésie bucolique. Le narrateur d'abord, bien qu'il se figure dans une scène de type urbain, joue depuis le début de l'idylle le rôle d'un chevrier. Les flûtistes qui divertissent Lycidas, quant à eux, sont des bergers

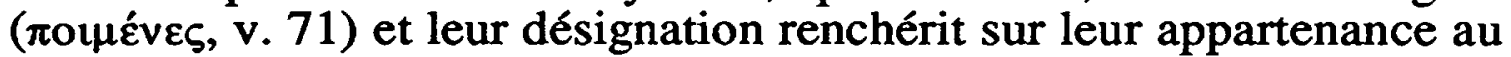
monde bucolique. Lycidas nomme le lieu dont ils tirent leur origine : le premier musicien est d'Acharnes (v. 71), que la tradition désigne comme le dème campagnard et rustique de l'Attique, où se pratique l'élevage ${ }^{60}$, le second «de Lycopé» (v. 72). On s'interroge deppuis les scholiastes de l'idylle 7 pour savoir s'il s'agit d'une localité d'Étolie ou de Cos ; la précision tire plus probablement son sens d'une allusion au bouvier Lycopas, évoqué dans la cinquième idylle ${ }^{61}$. Lacon souhaite en effet l'arbitrage de Lycopas dans la querelle poétique qui l'oppose à Comatas. Lycopas représente donc une autorité en matière de poésie bucolique. Tityre (v. 72), qui doit chanter l'histoire de Daphnis, apparaît exclusivement comme poète dans l'idylle 7, mais son nom est aussi celui du chevrier auquel le locuteur de l'idylle 3 confie ses chèvres quand il part faire sa cour à Amaryllis ${ }^{62}$. Daphnis, dont la légende constitue le sujet de la chanson de Tityre, est un bouvier. Désigné de façon presque formulaire : $\Delta \alpha \dot{\alpha} \varphi v i \varsigma$ ò ßoú $\alpha_{\varsigma}(v .73)$, il fournit encore la matière du poème de Thyrsis dans l'idylle 1 (v. 64-142) et intervient à

58 Voir E. L. BowiE, op. cit., p. 72-77. La même raison serait à l'origine de la situation de la Pastorale de Longus dans lîle de Lesbos. Mytilène est aussi la ville des parents de Daphnis et Chloé.

59 E. L. BOWIE, op. cit., p. 77.

60 Voir notamment ARISTOPHANE, Les Acharniens.

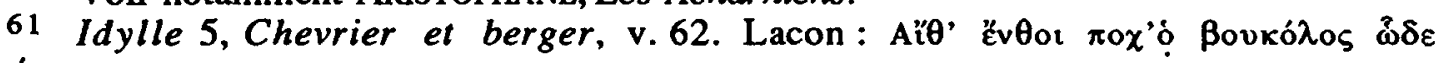

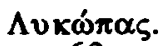

62 Idylle 3, La visite galante, v. 1-5. 
plusieurs reprises comme un critère de comparaison et d'appréciation de soi pour les personnages bucoliques des Idylles ${ }^{63}$. Il est aussi connu pour être l'amant de Pan lui-même ${ }^{64}$. C'est donc un personnage bucolique exemplaire. Comatas (v. 83), enfin, est un chevrier. Dans l'idylle 5, le personnage qui porte son nom accuse même Lacon de lui avoir volé une vóxos (v. 2), comme celle que revêt Eumée dans le chant XIV de l'Odyssée (v. 530).

Dans le poème de Simichidas, la référence à Pan est explicite, puisque le nom du dieu est cité au vocatif (v. 103) et qu'un passage du poème s'adresse directement à lui (v. 103-114). Simichidas sollicite l'aide de Pan pour gagner Philinos à Aratos. L'intervention de Pan dans une demande de ce type ne doit pas surprendre. Comme Aphrodite, a laquelle il est souvent associéc5, Pan «sait tromper, exciter le désir, veiller à l'union des sexes» ${ }^{66}$. Tous deux participent, parmi les divinités mythologiques, de valeurs de fécondité et de chasse qui les rapprochent. L'adresse à Pan est d'ailleurs suivie d'une invocation à Aphrodite (v. 115-119).

Les deux textes laissent cependant voir des contradictions et des dissonances qui écartent l'hypothèse d'un pur et simple changement de patronage. Ils confirment et développent l'ambivalence déjà latente dans le reste de l'idylle.

Les poèmes de Lycidas et Simichidas incluent des passages composés d'allusions géographiques (chez Lycidas les vers 74 à 77, chez Simichidas 111 à 116) dont le caractère érudit a été souligné. Cette caractéristique n'est pas leur seul point commun. Les deux textes évoquent des régions extrêmes et négativement connotées. Lycidas mentionne les monts Hémus, Athos, Rhodope et le Caucase, quand il décrit la désolation de Daphnis : ce dernier se consume en vain pour Xénéa comme la neige au pied de ces montagnes. Simichidas, quant à lui, menace Pan, s'il ne favorise pas les amours d'Aratos, de hanter la montagne des Édoniens en Thrace et l'Hèbre, face à l'Ourse polaire. Ce sont les mêmes régions que celles mentionnées dans le poème de Lycidas. Simichidas ajoute à ce paysage-repoussoir les terres désertiques et torrides des Ethiopiens, outre les cataractes du Nil, quand il envisage comme châtiment pour Pan une transhumance pervertie (v. 111-114), qui n'est plus l'occasion d'aller du plus chaud, la plaine, au plus frais, la montagne, mais mène à un surcroît de chaleur. Cet

63 Notamment idylle 5, v. 20 et 80.

64 Voir THÉOCRITE, Ep. 3; Anthologie Palatine, VII, 535 (Méléagre) et P. BORGEAUD, op. cit., p. 95 et 120 .

65 P. Borgeaud, op. cit., p. 101, 117-118, 159, 199, 202-204, 247-248.

66 P. BORGEAUD, op. cit., p. 118. 
univers excessif est opposé à la Sicile natale de Daphnis ${ }^{67}$, suggérée par le nom du fleuve Himéras, et aux côtes de Carie, évoquées à travers leurs sources. On peut alors remarquer que ce monde mauvais et violent est le cadre ordinaire de Pan : "A Pan conviennent ce que les Grecs appelaient les $\dot{\sigma} \sigma \chi \alpha \tau \iota \alpha i$, les "extrémités"», écrit P. Borgeaud"8. L'Arcadie elle-même se signale par ses montagnes et ses sécheresses ${ }^{69}$. Cependant, dans les Thalysies, Pan lui-même et un des personnages qui lui sont le plus proches, Daphnis ${ }^{70}$, sont représentés en victimes de cet univers. On observe dans les poèmes de Lycidas et Simichidas la volonté de souligner l'aspect négatif du paysage géographique symbolique des pouvoirs du dieu, opposé à des terres fertiles, traversées de fleuves. C'est une façon subtile et détournée de pointer sur les dangers qu'incarne Pan, et en particulier la stérilité.

Le patronage de Pan, si l'on examine de manière précise les deux poèmes, apparaît en effet systématiquement sous un jour défavorable, ou lié au thème de la stérilité. $P$. Borgeaud a montré ${ }^{71}$ que le rituel des scilles (v. 107) a lieu dans des conditions de pénurie alimentaire particulières. La mention des orties qui démangeront Pan (v. 109-110) joue à la fois sur l'image de la brûlure de l'amour insatisfait et sur l'existence d'une pratique pastorale pour stimuler la fécondité des chèvres. Il s'agit donc d'une parade à un échec. Pan, dans les poèmes de Lycidas et Simichidas, est également impuissant à satisfaire les demandes amoureuses de Daphnis et d'Aratos, ce qui fait écho à la renommée du dieu lui-même. Son ardeur est aussi légendaire que ses faillites, souvent expliquées par le fait que les possibilités d'assouvissement du chevrier, vivant solitaire dans ses montagnes, sont infimes. Chez Daphnis, le bien-aimé de Pan, l'échec amoureux conduit au silence poétique. Chaque fois que les Idylles le mettent en scène, il refuse de chanter $^{72}$; sa réputation de musicien exceptionnel, les plaintes que son malheur arrache à la nature entière, font ressortir la force de ce mutisme poétique. Or, dans les poèmes de Lycidas et Simichidas, l'absence de l'amant, pour Agéanax, ou son indifférence, pour Aratos, sont au contraire saisies comme des occasions de se consacrer à la poésie. Le

67 Voir aussi Idylle 1, Thyrsis, v. 125.

68 Op. cit., p. 94. Sur cette question, voir p. 94-95.

69 Voir P. BORGEAUD, op. cit., p. 15-16.

70 Les montagnes et les chênes qui pleurent Daphnis appartiennent au décor symbolique de Pan. Voir la présence des mêmes éléments et de la même structure dans le chant de Thyrsis, Idylle 1. Pour la relation entre le décor évoqué dans cet épisode légendaire et Pan, voir P. Borgeaud, op. cit., Les sentiers de Pan, p. 73-194 et K. LEMBACH, Die Pflanzen bei Theokrit, Heidelberg, Carl Winter Universitätsverlag, 1970.

71 Pour ce qui suit, consulter son analyse à propos du poème de THÉOCRITE, op. cit., p. 106-114.

72 Voir notamment l'idylle 1, Thyrsis, v. 64 à 142, la chanson de Thyrsis. 
silence de Daphnis paraît conditionné par ses rapports avec Pan. L'exemple de Daphnis met en évidence que le monde bucolique symbolisé par Pan est un monde sans alternative, sans issue, invivable. L'insatisfaction de Daphnis ne lui laisse pas d'autre solution que la mort, alors qu'Aratos, inscrit dans un contexte urbain, même s'il est placé sous la protection de Pan, a la ressource de tourner les talons et de participer à un banquet où l'on chantera de la poésie. Le monde qu'habite Pan n'offre pas une telle ressource. Pan est toujours en opposition au monde de la cité ${ }^{73}$, or l'idéal d'ó $\sigma v \chi i ́ \alpha$ du poème de Lycidas est un idéal urbain. La poésie, dans les Thalysies, est présentée comme une activité sociale; sa littérarité même implique une communauté humaine, des échanges ${ }^{74}$, qui nient qu'on puisse raisonnablement prétendre l'ancrer dans l'univers qui est celui de Pan. Il est, dans les poèmes de Lycidas et Simichidas, volontairement montré sous son jour le plus hostile et le plus inhumain, pour éveiller la vigilance du lecteur.

L'insistance des deux poètes, et en particulier du chevrier Lycidas, sur l' $\alpha \sigma v \chi i ́ \alpha$ marque le refus de la «panique» et de l'enthousiasme du dieu. Lycidas est installé sur sa couche, immobile. Aucun rapport entre son attitude et l'instabilité, l'agitation, de son représentant générique. Pan est une convention poétique, au même titre que les Muses. Simichidas s'adresse directement à lui, après avoir placé Lycidas sous la

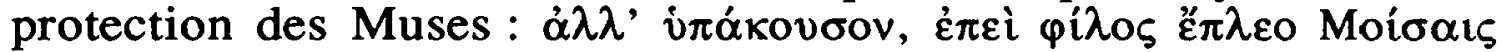
(v. 95). Ce changement de contexte est exposé à un dysfonctionnement puisque les Muses sont, depuis la Théogonie (et peut-être par opposition à Pan qui contredit l'harmonie et l'ordre auxquels elles prétendent et qui cherche, à travers les Nymphes, à les violer), hostiles aux chevriers qu'incarne Lycidas. Il signifie l'arbitraire de la référence aux dieux. Le monde bucolique est un décor, où se promènent des figures de comédie. Par leurs contradictions internes, en refusant de choisir entre l'alternative d'un monde entièrement dévolu à Pan et à la pastorale ou le monde urbain, en glissant sans cesse d'un registre à l'autre, les poèmes de Lycidas et Simichidas suggèrent au lecteur que la poésie bucolique est une combinaison, artificielle, ironique et savante, qui joue des confusions possibles. Il s'agit d'un lieu de synthèse, sélectionnant et acclimatant à ses propres usages, des données qu'elle tire de genres distincts.

L'aventure de Comatas en fournit l'exemple. Elle permet d'observer une résolution harmonieuse des postulations contradictoires exprimées jusqu'ici. Le personnage de Comatas unit le double patronage

73 Voir P. Borgeaud, op. cit., p. 95, 130-131, 258-261.

74 Autant de notions qu'on retrouve dans l'insistance des personnages à évoquer le poids de la réputation orale d'un auteur. 
des Muses et de Pan. La légende veut en effet que Comatas ait été puni par son maître parce qu'il distrayait ses chèvres pour les offrir aux Muses ${ }^{75}$. Lycidas dit explicitement que les abeilles viennent nourrir Comatas parce que la Muse a versé sur ses lèvres du «nectar», à la fois vin des dieux et miel (v. 82). Les abeilles reprennent en cela la fonction de nourrice qu'elles ont assumée pour Zeus et font ainsi de Comatas l'un des Olympiens, nourri du miel-ambroisie qui vient des Hespérides ${ }^{76}$. Dans l'idylle 5 , le personnage qui porte le nom de Comatas revendique dès les deux premiers vers des chants amœbées qu'il entame avec Lacon d'être le favori des Muses (Taì Moî $\sigma \alpha \imath$

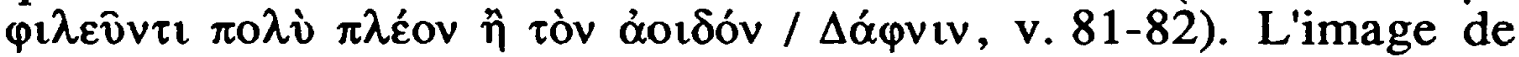
Comatas, cependant, est aussi saturée de références à Pan. Le poète est un chevrier : à ce titre son patron naturel est Pan. Le coffre dans lequel Comatas est enfermé est en cèdre; le poète compose sous un pin et une variété de chêne (v. 88). Les arbres ont une portée symbolique ${ }^{77}: \mathrm{P}$. Borgeaud montre que ceux qui entourent Comatas dénotent l'influence de Pan $^{78}$.

Comatas est une figure syncrétique. La notation du miel sur ses lèvres peut faire allusion à la syrinx de Pan. En effet, les chalumeaux sont liés ensemble par de la cire ${ }^{79}$, qui parfume l'instrument et la bouche du musicien. Ce phénomène se trouve à l'origine de la comparaison du son qui s'échappe de la flûte avec du miel liquide ${ }^{80}$. Or, à la fin de son poème, Lycidas qualifie Comatas en ces termes : $\dot{\alpha} \delta \dot{\nu} \mu \varepsilon \lambda \iota \sigma \delta o ́ \mu \varepsilon v o \zeta$ (v. 89). Ce verbe s'applique aussi bien à la musique des sons que des mots et entretient l'ambivalence. On assiste alors à une fusion entre le miel des Muses et l'art du dieu Pan. Or Comatas a la spécificité de donner l'exemple d'un poète fécond, alors que les autres poètes déclarés de l'idylle 7 (Daphnis, Tityre et Aristis) ne sont pas clairement

75 Voir G. WOJACZEK, Daphnis. Untersuchungen zur griechischen Bukolik, Meisenheim, A. Verlag, 1969 (Beiträge zur klassischen Philologie, 34), p. 46.

76 Voir P. Borgeaud, op. cit., p. 68 et A. B. CooK, The Bee in Greek Mythology, dans J.H.S., 15 (1895), p. 1-24.

77 Elle est clairement énoncée dans les Idylles. Dans l'idylle 5, les arbres sont l'enjeu de la rivalité de Lacon et Comatas, qui les nomment tour à tour comme pour s'approprier leurs vertus. On trouve aussi une confirmation des ces équivalences métaphoriques dans l'idylle 1: au moment de mourir, Daphnis, en guise de protestation, forme le vœu d'un renversement de l'ordre du monde et de son sens (v.132-136). Il imagine alors que les buissons portent des violettes, les pins des poires, de la même façon que les hiboux rivaliseraient avec les rossignols. Voir aussi les classifications et les conclusions de $\mathrm{K}$. LEMBACH, op. cit.

78 Voir op. cit., pour le pin p. 123 et 145, pour le chêne p. 21 et 30-32.

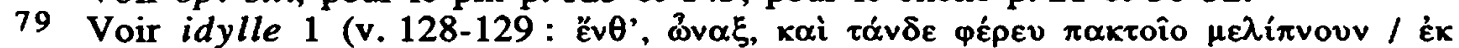

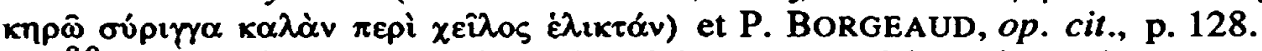

80 De là vient la métaphore du miel comme poésie, qu'on voit notamment à la fin de l'idylle 1 (v.146-147). 
représentés dans l'exercice de leur don. Son maître enferme Comatas pour provoquer l'intervention des Muses (v. 79) : la venue des abeilles et la mention du miel sur la bouche du poète sont les symboles du succès de cette ruse, de la faculté poétique dont est doué le poète. L'épisode de Comatas constitue aussi une allusion à la légende qui entoure la naissance de Pindare, où les abeilles déposent le miel de l'inspiration sur les lèvres du nourrisson. Ce récit est parfois situé pendant l'adolescence de Pindare et «dans le contexte théoleptique du midi ${ }^{81}$, qui se trouve être celui de l'idylle 7 . Cette légende appartient à la «geste» de la plupart des grands poètes ${ }^{82}$. Poète exemplaire, Comatas possède de nombreuses ressemblances avec Lycidas lui-même : il est

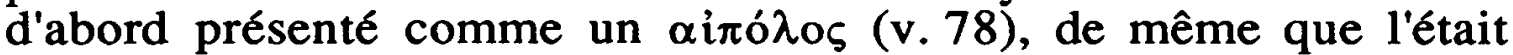
Lycidas (v. 12-19) ; sa faculté poétique n'est mentionnée qu'à la fin du

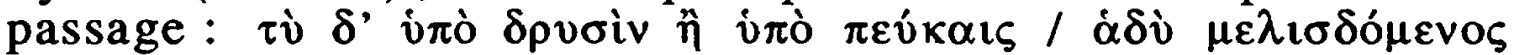

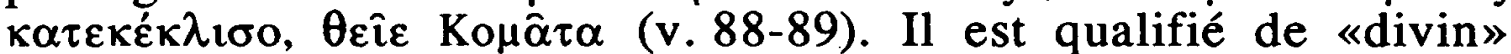
(v. 89) : Lycidas était décrit de façon à rappeler au lecteur l'apparition des dieux chez Homère. Lorsque Lycidas l'imagine à l'œuvre, c'est aussi dans une attitude nonchalante et agréable qui fait écho à la sienne pendant l'absence d'Agéanax. Comatas donne donc lieu à l'expression d'un art poétique métaphorique qui vaut pour l'ensemble de l'idylle.

Comatas est enfermé dans un coffre : il est nécessaire de s'interroger sur cette aventure insolite. Dans l'idylle 1, parmi les représentations qui ornent la coupe que le chevrier se propose d'offrir à Thyrsis, s'il se montre bon chanteur, figure un enfant absorbé à tresser un filet pour prendre des sauterelles (v. 45-54). Les sauterelles, à examiner leurs occurrences dans le corpus des Idylles et dans l'idylle 7 (v. 41), sont une métaphore du poète. On retrouve donc l'idée du poète mis en cage. L'image purement animale des idylles 1 et 5 étant incarnée, dans l'idylle 7 , dans la figure de Comatas. La relation entre les deux textes est d'autant plus forte que le décor où est placé l'enfant de l'idylle 1 fusionne des éléments présents dans les idylles 7 et 5 , révélant un schéma récurrent chez Théocrite. On retrouve les pierres sèches (1, v. 47) du début de l'idylle 7 (v. 22), la vigne (1, v. 45-46) évoque le vin que boivent Lycidas dans sa chanson $(7, v .65,69,70)$ et Simichidas à la fin du poème $(7$, v. $147,154-155)$.

Il y a débat pour savoir si la vigne de l'idylle 5 est sèche ou mûre, comme dans l'idylle 1 . Dans les deux cas, il importe de remarquer que Comatas invite les sauterelles à s'en désintéresser (v. 108-109), de même que l'enfant de l'idylle 1 ne s'occupe pas des ravages qu'y font les renards. On observe aussi dans l'idylle 7, passée l'évocation

81 Voir P. BORGEAUd, op. cit., p. 254, n. 6.

82 Homère, Hésiode, Sophocle, Platon, Virgile, Lucain et Ambroise. Voir A. B. CoOK, op. cit., p. 7-8. 
symposiaque du vin, où sa valeur sociale est dominante, une insistance très nette au profit des sources d'eau vive ${ }^{83}$. Le vin est une métaphore courante de la poésie. On doit en conclure que les idylles 1,5 et 7 opposent deux esthétiques à travers l'image des poètes-sauterelles, voués à l'enfermement, et celle des vignes qui les entourent. Le vin est avant tout dilapidation, oubli à soi-même, profusion généreuse et incontrôlée, alors que les épreuves de Comatas enfermé dans son coffre sont désignées par le verbe $\dot{\varepsilon} \kappa \pi$ ové $\omega-\hat{\omega}$ (v. 85), que Lycidas emploie également pour signifier un travail littéraire minutieux et attentif (v. 51). Cette dernière conception, faite de contrainte et de vigilance, est proposée comme le meilleur moyen d'accéder à la création. L'exemple de Comatas suggère que, plus nombreuses et sévères sont les exigences auxquelles le poète est soumis, par le recours à des genres ou des

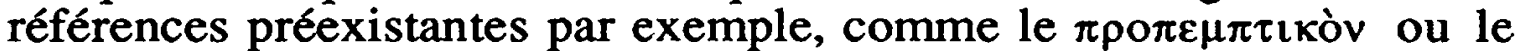
$\pi \alpha \rho \alpha \kappa \lambda \alpha v \sigma i \theta u \rho o v$, ou à des formes brèves telles que l'idylle et l'épigramme, plus il fait preuve d'ingéniosité et de virtuosité. La réclusion et la fécondité de Comatas sont le contrepoint de l'errance et de la stérilité de Daphnis dans les idylles 7 et 1 . Comatas est une figure harmonieuse de poète, quoique placé sous les auspices de Pan, parce que son emprisonnement est une parade à la dilapidation. Le miel, dans son cas, n'est pas seulement séduction qui s'échappe ${ }^{84}$, il nourrit matériellement. Dans la conclusion de l'idylle 1 , le chevrier joue encore de cette double valeur possible :

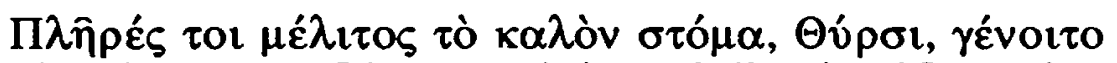

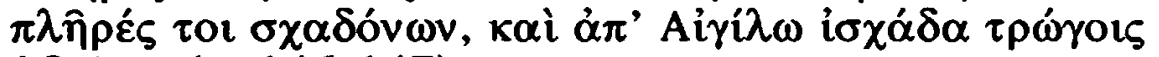

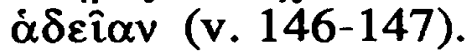

Les rayons de miel s'opposent au miel liquide qui s'échappe de la syrinx. Contrainte et miniature forment deux ressources majeures de l'idéal poétique qu'évoque Théocrite. Le poème de Lycidas suggère avec virtuosité, par allusion, par variations et échos à partir de textes et de légendes antérieures, voire à partir de son propre recueil, la conception d'une poésie savante, qui s'impose toutes sortes de contraintes pour atteindre à plus de finesse et de raffinement.

Les renards mentionnés dans les idylles 1 et 5 permettent de compléter cet art poétique. Dans l'idylle 1 , le premier pille les raisins de la vigne, tandis que le second rôde autour de l'enfant pour s'emparer de son déjeuner. Dans l'idylle 5, Comatas exprime sa haine et son mépris

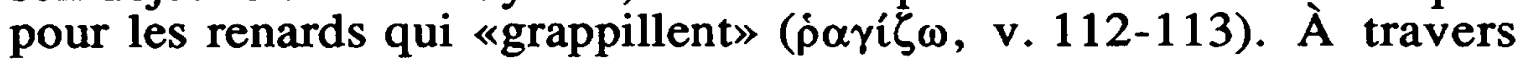

83 Sources Bourina (v. 6), Hyetis (v. 115), Oikous (v. 116), l'eau sacrée des Nymphes (v. 136) et les fontaines de la fin du texte (v. 142).

84 Voir P. BORGEAUd, op. cit., p. 128. 
l'image des renards, Théocrite désigne ce que ne doit pas être la création poétique, un pillage et une exploitation maladroite, sans originalité, de ses modèles. Le poète enfermé ne procède pas à l'assemblage de parties empruntées à autrui. Il trouve en lui seul ses ressources. Il tire de l'ancien un neuf véritable. L'image des renards pillant des grappes de raisins rappelle aussi la fable d'Esope Le renard et les raisins ${ }^{85}$. Elle raconte précisément l'échec du larcin, l'incapacité du renard à se hausser jusqu'aux grappes convoitées. La morale blâme qui préfère déprécier ce qu'il n'a pu obtenir, plutôt que de reconnaître sa faillite. Le poème de Lycidas sous-entend ainsi que les critiques qu'on pourrait adresser à sa poétique tiennent à la jalousie des poètes traditionnels, incapables d'atteindre le même résultat que lui. Il revendique pour sa poétique d'être une interprétation de la tradition, seul moyen de la continuer. L'évocation de Comatas, ramassée en quelques vers, dans une vignette, à l'intérieur du poème de Lycidas, au centre de l'idylle 7 , permet d'exprimer un idéal poétique de concision où les Muses et Pan sont unis. Le thème de la stérilité panique y est conjuré par l'idée de la contrainte et de la restriction, de la discipline, qui gouvernent le poète. Dans l'ordre de la symbolique végétale, cet équilibre original appelle le patronage d'une nouvelle divinité, Déméter.

L'idylle 7, par son titre, Les Thalysies, annoncées comme une fête des aires (v. 3 et 31 à 34), et par l'importance dévolue dans sa conclusion au thème des fruits, insiste sur l'idée de fécondité, que ni les Muses ni Pan ne parvenaient à satisfaire ou à figurer. Pour qualifier les poèmes des auteurs dont il critique l'esthétique, Lycidas emploie l'adverbe $\dot{\varepsilon} \tau \omega ́ \sigma \iota \alpha$ (v. 48), qui signifie au sens propre «infructueux». Déméter, déesse de la terre et de la moisson, apparaît alors comme une divinité de substitution et de fusion particulièrement indiquée. Ses rapports avec Pan sont établis ${ }^{86}$, mais contrairement à ce dernier elle incarne la culture, par opposition au sauvage, la fécondité maîtrisée, par opposition à la dilapidation. Déméter, dans un contexte poétique où le rôle de la littérarité a été souligné, est aussi typiquement celle qui enseigne. Elle trouve donc à juste titre sa place à la fin du poème. Si aucun instant du rituel des Thalysies n'est décrit, si l'on passe tout de suite à l'évocation du banquet qui suit la célébration religieuse, si, hormis dans le dernier vers (v. $157: \delta \rho \alpha ́ \gamma \mu \alpha \tau \alpha$ et $\mu \alpha \kappa \omega v \alpha \varsigma$. K.

85 «Un renard affamé, voyant des grappes de raisins pendre à une treille, voulut les attraper ; mais, ne pouvant y parvenir, il s'éloigna en se disant à lui-même : 'c'est du verjus'. Pareillement certains hommes, ne pouvant mener à bien leurs affaires, à cause de leur incapacité, en accusent les circonstances», ÉsOPE, Fables, 32, traduction É. CHAMBRY, Paris, PUF, 1927.

86 Voir P. Borgeaud, op. cit., p. 31-32. 
Lembach ${ }^{87}$ montre qu'il s'agit d'attributs de Déméter), aucune plante en relation avec la moisson n'est mentionnée, c'est que Déméter et les Thalysies ont une valeur bien plus métaphorique, comme l'a vu $F$. Lasserre, que réaliste. On peut remarquer que le nom $\dot{\alpha} \lambda \omega \alpha ́$ (v. 34), employé pour expliquer la fête des aires que sont les Thalysies, à l'origine de la formation de l'épithète de Déméter ('A $\lambda \omega \hat{i} \delta o s$, v. 155), est aussi utilisé dans l'idylle 1 pour désigner la vigne près de l'enfant ${ }^{88}$. Les deux textes entrecroisent leurs réseaux symboliques et s'enrichissent réciproquement. Les références concrètes et l'humour, analysés par G. Giangrande, qu'impliquent ces poèmes, existent, mais ils ne forment qu'une composante, qu'une tonalité, d'une œuvre qui s'emploie à multiplier les niveaux de signification et de lecture. Le poème de Théocrite résume dans un bouquet final l'ensemble des préceptes et des attitudes qu'il a pu évoquer.

La référence à Déméter loin de se trouver exclusivement liée aux travaux agricoles ou à un rituel religieux est riche d'échos littéraires. E. L. Bowie a fait remarquer ${ }^{89}$ que la place accordée dans les Thalysies à Déméter pouvait s'expliquer par allusion à la Déméter de Philétas, ancêtre du genre bucolique. Callimaque fait aussi allusion à ce textes . Nombreuses, enfin, sont les réminiscences d'Hésiode dans les Thalysies. Or Déméter est une des divinités principales des Travaux et des Jours. Si Théocrite, à travers Simichidas, exprime son refus d'un patronage des Muses qui prive le poète de son initiative et ne reconnaît pas la part du travail individuel, il est intéressant de se reporter aux vers 298 à 301 des Travaux: Hésiode exhorte Persès au labeur pour se concilier les bonnes grâces de Déméter, qui remplira alors son grenier. Suit un long éloge du travail (v. 302-316), qui n'est pas dénué d'intérêt par rapport au propos de l'idylle 7 elle-même. L'idée de la faim, châtiment de celui qui ne travaille pas, fait écho aux chants «infructueux» (v. 48) dont parle Lycidas et contraste avec la présentation d'un poète repu et bienheureux que répète l'idylle 7 : Lycidas dans l'attente d'Agéanax, Comatas nourri par les abeilles et Simichidas chez Phrasidamos et Antigénès. Ce thème s'épanouit dans la conclusion de l'idylle, qui s'achève sur le spectacle d'un banquet et d'une nature regorgeant de fruits. L'expression dont Simichidas usait pour se

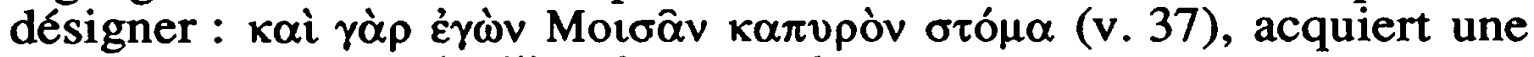
pertinence toute particulière dans un tel contexte.

Mais on peut aller plus loin et avancer que le parallélisme entre l'idylle 7 et le texte d'Hésiode vient renforcer la conception du poète

\footnotetext{
87 Voir op. cit, p. 162-163.

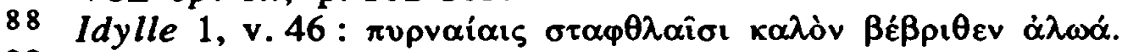

89 Op. cit., p. 80.

90 Aetia, fr. 1.9 et suivants.
} 
comme artisan, exerçant une $\tau \dot{x} \chi \vee \eta$, qui s'exprimait dans la comparaison avec l'architecte et l'utilisation du nom $\tau \dot{\varepsilon} \kappa \tau \omega v$. À l'image du poète inspiré, traversé par le souffle de la Muse, qu'on observe au début de la Théogonie, par exemple, Théocrite substitue l'idée d'un savoir et d'un savoir-faire comparable à celui que Déméter exerce sur la terre et sa culture. La relation verticale allant de la divinité au poète est effacée au profit d'une identité et d'une complicité qu'illustre un sourire partagé. Le même verbe $\gamma \varepsilon \lambda \alpha ́ \sigma \sigma \alpha \imath$, symbolique d'une attitude divine, est employé pour caractériser celle de Lycidas (v. 42), puis de Déméter (v. 156). Rétrospectivement, la présentation de Lycidas comme un dieu s'avère moins ironique que remplie d'humour, venant tempérer la gravité du discours que le poème développe ensuite.

On assiste, dans les vers 128 à 157 , sous l'autorité de Déméter, à une réconciliation générale, voire une fusion, des éléments emblématiques de Pan et des Muses. Leur opposition se résout en combinaison. Désormais, les Nymphes, liées à Pan, sont dites Castalides (v. 148). Une place leur est explicitement faite dans la tradition poétique apollinienne des Muses, dont la présence est renforcée par la mention du Parnasse (v. 148). Les Nymphes unissent Phébus et Pan, opposés dans les poèmes de Simichidas et Lycidas. Le personnage de Simichidas lui-même subit une légère altération. L'analyse des préférences sexuelles des différents personnages de la poésie bucolique révèle que le chevrier, «bon» poète bucolique, est homosexuel, tel Lycidas, tandis que le bouvier est hétérosexuel ${ }^{91}$, comme Daphnis et Simichidas lui-même (v. 96). Or Daphnis, amoureux d'une femme, est incapable de chanter dans le poème de Lycidas (v. 73-77) : il se dissout, disparaît, tandis que seuls se font entendre la montagne et les chênes. Simichidas, dans sa chanson, se donne pour l'amant de Myrto,

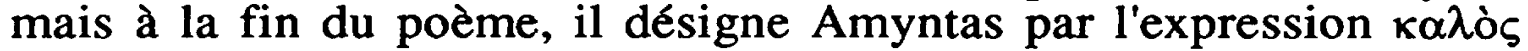

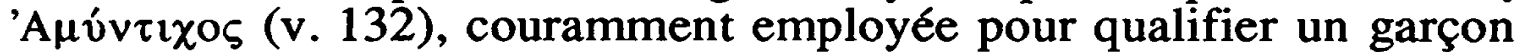
qu'on désire, en particulier dans les graffitis ${ }^{92}$. En payant ce tribu à Amyntas, qui ne sort pas du rôle de personnage muet, Simichidas trahit un intérêt qui le rapproche de la figure du chevrier-poète par excellence et l'éloigne du personnage entièrement ironique qu'il avait jusque-là assumé. Tous les éléments participent ainsi d'une profonde cohérence symbolique.

On a signalé un jeu, au cours du poème, entre les valeurs représentées par le vin et l'eau. A la fin de l'idylle 7, Simichidas parle de

91 Voir D. M. HAlperin, op. cit., p. 183-184 et B. A. VAN Gronigen, op. cit., p. 134. Voir aussi le personnage de Gnathon dans la Pastorale de Longus.

92 Voir Callimaque, fr. 73 Pf.; Gow-Page, Greek Anthology of Hellenistic Epigrams, v. 760 et E. ROHDE, Griech. Rom. ${ }^{3}$, p. 173, n. 1. 


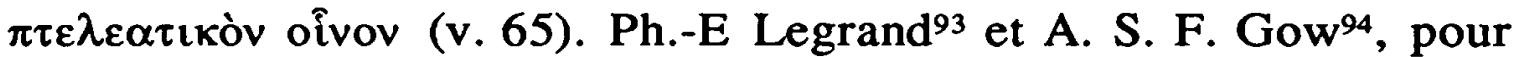
expliquer cette expression, se réfèrent à une scholie selon laquelle il s'agirait d'un cru particulier, peut-être de Cos. Il semble plus éclairant de suivre E. L. Bowie ${ }^{95}$ et de rapprocher cette formule des $\pi \tau \varepsilon \lambda \varepsilon \dot{\varepsilon} \alpha \iota$ évoqués vers 8 et 136, en liaison avec les sources Burina et l'eau sacrée qui s'échappe d'un lieu consacré aux Nymphes. Le vin auquel il est fait allusion a essentiellement une valeur symbolique. Par la médiation d'arbres présents dans un site où se trouve l'eau caractéristique des Nymphes (il est interdit de leur faire des offrandes de vin ${ }^{96}$ ), le vin luimême participe désormais de l'univers bucolique, sans la notion de violence et d'excès qu'il comporte dans le culte de Pan $^{97}$. Enfin, dans les vers 150 à 155, une comparaison associe ce vin au nectar : le nectar est la boisson des dieux, mais également le terme employé pour caractériser le miel sur les lèvres de Comatas. Chacune des valeurs possibles entre en contact. L'univers poétique de la conclusion de l'idylle n'est plus marqué par aucune sorte de déchirement ou de rupture entre des postulations contraires.

Une étude des plantes mentionnées dans l'idylle 7, fondée sur les classifications qu'opère $\mathrm{K}$. Lembach, montre que la fusion des symboles concerne aussi cet aspect du poème. Plusieurs séquences se sont succédé jusqu'à présent, chargées de connotations distinctes. Les plantes évoquées au début du texte (peuplier, ormes et bocages du vers 8) annoncent celles que le lecteur retrouve à partir du vers 136 , dotant le poème d'une structure circulaire qui doit convaincre de la rigueur de sa construction et de sa valeur démonstrative. Par l'ombre et l'eau qui les environnent, ces arbres pointent l'univers pastoral, sans en être des symboles avérés. Au cours des vers 63 à 68 , quand Lycidas s'imagine célébrant la traversée d'Agéanax, interviennent des plantes caractéristiques de l'univers pastoral (conyze, asphodèle et ache frisée), mais les fleurs (aneth, roses, giroflées blanches) sont en nombre supérieur. Or ces dernières, dans le système de référence de la poésie grecque, sont plus la marque d'un souci urbain qu'une caractéristique du monde bucolique. La tonalité du passage reste ambivalente. C'est seulement à la fin de son poème (v. 81-88) que Lycidas évoque des arbres qui font vraiment référence à Pan (cèdre, pin et yeuse), lorsqu'il décrit, entre Comatas et lui, une scène d'un fort investissement symbolique.

93 Bucoliques grecs, Paris, Belles Lettres, 1972, p. 11, n. 2.

94 Theocritus, ed. with a Translation and a Commentary, Cambridge, Cambridge University Press, 1952.

95 Op. cit., p. 79.

96 Voir P. BORGEAUD, op. cit., p. 240.

97 Voir P. BORGEAUD, op. cit., p. 32 et 240. 
Du vers 136 jusqu'à la fin du texte, chacune de ces plantes et chacune de ces tonalités sont à nouveau conviées et cette fois fusionnées. Le passage de l'idylle 7 que l'on considère donc comme l'évocation bucolique la plus achevée est en réalité celui où se mêlent tous les courants possibles déjà envisagés, auxquels s'ajoute une dernière variété : les fruits (poires, pommes, prunes). Ils sont évidemment en rapport avec la célébration des Thalysies, mais aussi avec le thème de l'accomplissement poétique. Le poème se conclut avec la mention des épis et des pavots (v. 157). Les premiers n'appartiennent pas aux registres étudiés par $\mathbf{K}$. Lembach. Les épis, emblèmes de Déméter, par opposition aux glands, sont le symbole du cultivé par rapport à l'aliment sauvage caractéristique de l'univers de Pan : ils rappellent une dernière fois la leçon du poème. Le pavot résume toutes les tendances : il est à la fois un symbole de Déméter, une fleur typique de la flore bucolique et une sorte de fruit, puisqu'il est comestible. La brassée évoquée par Simichidas réunit toutes les nuances précédentes. De surcroît, dans une pointe finale, l'oubli dont s'accompagne l'absorption du pavot fait écho au détachement de l'ó $\sigma v \chi i ́ \alpha$ permise par

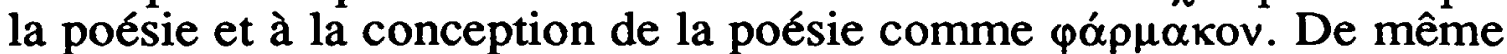
que le décor de la scène mêle la grotte du monde de la mythologie panique $^{98}$ à une nature civilisée, un locus amonus qui exprime une nostalgie de citadins, la dernière image du poème convie simultanément plusieurs symboles pour figurer un univers artificiel et original.

L'étude des animaux cités dans le poème révèle d'abord l'étonnante absence de tout bétail caractéristique de la pastorale : un bouc est évoqué v. 15, mais seulement parce que sa peau recouvre les épaules de Lycidas ; les chèvres du v. 87 n'existent que dans l'imagination du narrateur. Toutes les bêtes citées ont, en revanche, une liaison évidente avec le chant et une valeur symbolique par rapport à la poésie (excepté le lézard auquel Lycidas compare Simichidas, v. 22) : volailles, coq (animaux de basse-cour, animaux domestiques) et grenouilles symbolisent la discordance, tandis qu'alouettes (v. 23 et 141), sauterelles (v. 41), chardonnerets (v. 141), tourterelle (v. 142), possèdent une valeur positive. Quoique silencieuses, les abeilles sont néanmoins un élément majeur des mythes qui ont trait à la poésie. On observe donc une pluralité exemplaire du poème, qui l'éloigne de la dénotation d'un genre particulier, et prouve son investissement poétique et sa construction argumentative. D'autant que sept types d'animaux sur quinze sont mentionnés en l'espace de dix-neuf vers (v. 124-143), dans la conclusion, au moment où leur nécessité stratégique est la plus forte. On note aussi une reprise des alouettes mentionnées au début du poème et des exemplaires abeilles, qui assurent au poème une continuité,

98 Voir P. BORgEAUd, op. cit., p. 68-69, 75-81, 233-234. 
comme la présence des personnages muets d'Eucritos et Amyntas. L'évolution dramatique se marque dans de discrètes variations. Ainsi

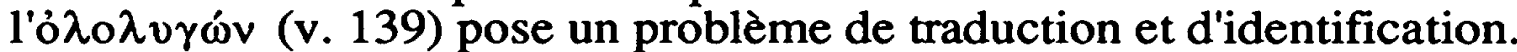
S'agit-il d'une grenouille, et d'une répétition par rapport au vers $41^{99}$, ou d'un animal différent, d'un oiseau ${ }^{100}$ ? $\mathrm{H}$. White propose d'y voir un rossignol ${ }^{101}$. La mention de l'ó $\lambda \circ \lambda v \gamma \omega \dot{v} v$ comme rossignol, qui symbolise un chant parfait ${ }^{102}$, traduit une réconciliation de l'auteur avec les images les plus conventionnelles de la poésie ${ }^{103}$, que le début du poème doit avoir appris à lire et utiliser.

On ne saurait trop insister sur la virtuosité des derniers vers qui ramènent l'inscription de l'écart et de l'humour à leur degré le plus vif. L'idéal de bien-être dépeint dans les vers 147 à 155 reste tout entier marqué par les propos ironiques prononcés par Lycidas (v. 24 à 26) au début de l'idylle ; rien ne dément dans le corps du poème l'interprétation de Simichidas comme un parasite ${ }^{104}$. Au contraire, le narrateur joue sur la dualité possible du sens. Les références aux personnages d'Héraclès et de Polyphème sont également ambiguës : sans doute la mention du sage Chiron et de Polyphème fait-elle allusion à un contexte épique, à travers Stésichore et Homère ${ }^{105}$, mais le verbe $\chi 0 \rho \varepsilon \hat{v} \sigma \alpha \iota \quad$ (v. 153), appliqué à Polyphème, appelle aussi la réminiscence d'Euripide ${ }^{106}$, où la scène a une valeur ironique. Enfin, le lecteur de l'idylle 7 ne peut pas ne pas penser aux idylles 13 et 11 , où il retrouve Héraclès et le Cyclope mis en scène d'une façon qui contraste avec la tonalité épique. Ces allusions mythiques sous-entendent tout un art du contraste et de l'allusion au service de l'humour, dont la position à l'extrémité du texte souligne l'importance décisive.

L'idylle 7 est une pièce parfaitement cohérente et achevée, qui propose au lecteur un mode d'appréhension et de compréhension du texte où le jeu des identifications littérales est dénoncé, au profit d'une perception des différences de ton et des variations internes de l'œuvre.

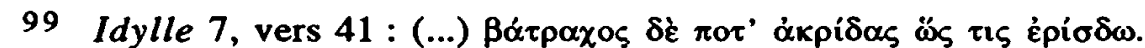

100 Voir P. ChantranNe, op. cit., article ò $\lambda_{0} \lambda v \gamma \omega \dot{v}$.

101 Studies in Theocritus and other Hellenistic poets, Amsterdam, J. G. Gieben, 1979, The Frog or the Nightingale ? A Theocritean problem, p. 9-16.

102 Il est intéressant de remarquer, dans le contexte de l'idylle 7 , que le chant du rossignol correspond, selon la légende, à la plainte que lui inspire la disparition d'Itys. On retrouve donc l'idée que l'achèvement poétique est obtenu à partir d'une douleur initiale, métamorphosée ou sublimée.

103 Voir G. Giangrande, op. cit., p. 491, n. 1 et Sympotic Literature and Epigram, Genève, Entretiens de la Fondation Hardt, 1968. Dans l'idylle 5, Comatas reprend ces images dans le dernier couplet des chants amœbées qu'il échange avec Lacon (v. 136-137).

184 Voir à ce propos l'analyse de G. GiangRANDE, op. cit., p. 503 et suivantes.

105 Odyssé, XI.

106 Le Cyclope. 
Loin d'être une forme codifiée ou une vision du monde, la poésie bucolique est fondée sur le mélange des genres, l'allusion et la dissonance ${ }^{107}$. L'extrême habileté du poète se marque dans son recours aux figures mêmes qu'il dénonce, dans son art de scènes assez typiques pour égarer si elles ne sont pas décryptées dans un perpétuel renvoi d'un point à l'autre du poème. Par l'ironie, des représentations, à l'époque de Théocrite peut-être encore seulement susceptibles d'être interprétées comme conventionnelles, sont cependant senties comme une menace et réactivées par le jeu des décalages.

Plus encore qu'un jeu de virtuose, les Thalysies se présentent comme une réflexion sur le statut et le travail du poète, alliant renouveau et exploitation des œuvres anciennes. Elles élaborent et illustrent une poétique. Il est impossible de s'en tenir à une lecture partielle de l'idylle 7 ou d'estimer qu'elle ne constitue qu'une rhapsodie de morceaux cousus ensemble indépendamment de toute logique profonde. Peut-être même l'observation de la construction et du fonctionnement de ce poème devrait-elle présider à une relecture de l'ensemble des Idylles.

Ecole normale superieure,

Laurence PLAZENET

rue d'Ulm 45,

F-75005 Paris.

107 Sur la technique allusive mise en œuvre par Théocrite, voir aussi la conclusion de G. Giangrande, op. cit., p. 521 . 